University of Nebraska - Lincoln

DigitalCommons@University of Nebraska - Lincoln

Faculty Publications, Department of Physics and Astronomy

Research Papers in Physics and Astronomy

October 2003

\title{
Controlling $\mathrm{H}^{-}$detachment with few-cycle pulses
}

S. X. Hu

University of Nebraska - Lincoln, suxing@lanl.gov

Anthony F. Starace

University of Nebraska-Lincoln, astarace1@unl.edu

Follow this and additional works at: https://digitalcommons.unl.edu/physicsfacpub

Part of the Physics Commons

Hu, S. X. and Starace, Anthony F., "Controlling $\mathrm{H}^{-}$detachment with few-cycle pulses" (2003). Faculty Publications, Department of Physics and Astronomy. 46.

https://digitalcommons.unl.edu/physicsfacpub/46

This Article is brought to you for free and open access by the Research Papers in Physics and Astronomy at DigitalCommons@University of Nebraska - Lincoln. It has been accepted for inclusion in Faculty Publications, Department of Physics and Astronomy by an authorized administrator of DigitalCommons@University of Nebraska Lincoln. 


\title{
Controlling $\mathrm{H}^{-}$detachment with few-cycle pulses
}

\author{
S. X. Hu* and Anthony F. Starace ${ }^{\dagger}$ \\ Department of Physics and Astronomy, The University of Nebraska-Lincoln, Nebraska 68588-0111, USA
}

(Received 26 June 2003; published 20 October 2003)

\begin{abstract}
We present a detailed analysis of short-pulse detachment processes using few-cycle pulses with the aim of demonstrating means for controlling such processes. We first generalize the standard Keldysh-type formalism for laser-target interactions (in which final-state interaction between the detached electron and the core is ignored) to include the possibility that the vector potential is nonzero at the end of the interaction between a short laser pulse and the target. With this formalism in hand, we examine the effects of half-cycle pulses (HCPs) on detachment of the prototypical negative ion $\mathrm{H}^{-}$, and show that detachment by pairs of oppositelydirected (i.e., "bidirectional") HCPs allows one to understand the interference pattern seen in detachment by single-cycle pulses. We also examine in detail the transition from few-cycle pulses to many-cycle pulses as various experimental parameters are varied, i.e., the laser frequency, the laser-pulse duration, and the absolute phase of the carrier wave with respect to the pulse envelope. Finally, we examine the use of pairs of singlecycle pulses, differing in phase by $180^{\circ}$, together with a modest static electric field to control coherently the extent of $\mathrm{H}^{-}$detachment as the delay between the pulses is varied. Our simulations show that this scheme allows one to modulate the $\mathrm{H}^{-}$detachment probability by $\sim 30 \%$, which is far higher than has been achieved for similar schemes using many-cycle pulses. Although our results are presented specifically for $\mathrm{H}^{-}$, they apply to detachment of any negative ion having $s$-state valence electrons; in addition, the qualitative information on half-cycle, single-cycle, and few-cycle pulse interactions should be generally applicable to short-pulse detachment or ionization of other target atoms or ions.
\end{abstract}

DOI: 10.1103/PhysRevA.68.043407 PACS number(s): 32.80.Qk, 32.80.Gc, 42.50.Vk, 32.80.Rm

\section{INTRODUCTION}

Rapid progress in the techniques for generating ultrashort laser pulses has enabled experimentalists to produce pulses containing only a few optical cycles $[1,2]$. Such few-cycle pulses may be focused to obtain intensities as high as $\sim 10^{16} \mathrm{~W} / \mathrm{cm}^{2}$. Since an ultrashort pulse may have only a few oscillations inside its pulse envelope, the absolute phase of the monochromatic part of the laser field with respect to its pulse envelope becomes an important part of the description of its interaction with matter [3-6]. Furthermore, if the number of laser oscillations inside the pulse is decreased to only a single cycle or less (e.g., a half cycle), it is an interesting question whether there are qualitative changes marking the interaction of such unusual pulses with matter. Halfcycle pulses (HCPs) can be taken as a limiting case. Experimentalists have already shown how to produce HCPs via short-pulse photoexcitation of a semi-insulating wafer $[7,8]$. Half-cycle pulses have been used extensively to ionize (kick) Rydberg atoms $[9,10]$, to measure the quantum phase of wave packets [11], and to study the dynamics of diamagnetic Zeeman states [12]. Since the Kepler period of a high$n$ Rydberg state may be much longer than the typical duration of a HCP, the impulse approximation has been used to describe the HCP's "kick" imparted to a slowly revolving Rydberg electron. However, the impulse approximation is no longer valid if the HCP duration is longer than the typical Kepler period of a system. One approach, of course, is to carry out a direct numerical integration of the time-

\footnotetext{
*Email address: suxing@lanl.gov

${ }^{\dagger}$ Email address: astarace1@unl.edu
}

dependent Schrödinger equation [13,14]. Alternatively, one may reexamine analytic Keldysh-type theories [15-22], which are well-established for the description of the interaction of intense monochromatic fields with matter. These have been generalized to the case of finite but long laser pulses having many cycles $[23,24]$; they have also been used to treat few-cycle pulses for the case that the time integral of the electric field of the pulse is zero $[25,26]$. The few-cycle case considered here requires further generalization in order to treat instances in which the vector potential no longer vanishes at the end of a few-cycle or half-cycle pulse $[27,28]$. Although this case has been recognized as unusual [29-32], it is becoming common now that experimentalists can produce true half-cycle pulses [7]. In addition, as we shall show below, analysis of the case of two oppositely polarized and time-delayed coherent half-cycle pulses provides insight into the unusual electron angular and momentum distributions produced in detachment of $\mathrm{H}^{-}$by singlecycle pulses.

The characteristics of few-cycle pulses have applications to quantum control of atomic processes. Coherent control of quantum systems has drawn increasing attention for more than 20 years [33]. The basis for quantum control is the fundamental principle of coherent state superposition in quantum mechanics, whereby the superposition of alternative transition amplitudes can lead to constructive or destructive quantum interference effects. Such interference effects may enhance or suppress a particular transition, depending on the relative phase difference of the alternative transition amplitudes. For example, recently a scheme for phase control of the transition yield of alkali atoms has been analyzed in which there is a dc field-induced interference between a resonantly enhanced two-photon transition and a single-photon (second harmonic) transition to the same final (continuum) 
state of an alkali atom [34]. Also, recently the phase difference between different final-state partial waves produced by a two-photon transition and the corresponding (coherent) single-photon (second harmonic) transition in the Rubidium atom has been measured [35]. Among the pump-probe types of coherent control processes [33] are those that employ a static electric-field to control the motion of electron wave packets produced either in highly excited Rydberg states $[36,37]$ or in $\mathrm{H}^{-}$photodetachment $[23,24]$. In the case of $\mathrm{H}^{-}$ photodetachment $[23,24]$, the basic idea is to use an external electric field to reflect one of the two outgoing electron wave packets produced by an initial laser pulse linearly polarized along the static field direction. (One wave packet accelerates away from the origin in the direction of decreasing static electric field potential energy; the second wave packet decelerates as it moves in the direction of increasing static electric field potential energy and is reflected by the potential.) When the reflected wave packet returns to the vicinity of the origin, a time-delayed second laser pulse (coherent with the first) produces another two oppositely moving electron wave packets, one of which will overlap and interfere with the reflected electron wave packet. By controlling the phase of the second laser pulse, one can produce either constructive or destructive quantum interference, thereby modulating the $\mathrm{H}^{-}$ detachment cross section. Obviously, the maximum possible modulation is about $50 \%$ in principle, because the static field only reflects one of the two outgoing wave packets produced initially. [In practice, the maximum modulation is usually considerably less owing to the spreading of the initial wave packet; e.g., in Refs. [23] and [24] (in the case in which only a static electric field was used) modulations of only $2 \%$ and $10 \%$ were obtained, respectively.] However, the situation may be changed qualitatively if few- or half-cycle pulses are employed: namely, one may be able to produce detached electron wave packets in only a single direction, thereby enabling one to increase the possible modulation of the $\mathrm{H}^{-}$ detachment cross section in a pump-probe control scheme such as that just described.

In this paper, we analyze theoretically the process of $\mathrm{H}^{-}$ detachment by half-cycle and few-cycle pulses using a generalization of the familiar Keldysh-type theory that includes the case of a possible nonzero vector potential at the end of the pulse. Our emphasis is on the analysis of quantum control of the $\mathrm{H}^{-}$detachment process by using half- or fewcycle pulses together with a static field. We demonstrate the possibility of modulating the $\mathrm{H}^{-}$detachment cross section by up to $\sim 30 \%$. We also demonstrate the essential role of the absolute phase of the few-cycle pulses. In particular, the symmetry of the photoelectron momentum spectrum is found to break down as the number of laser cycles in the pulse becomes of order 1 . The dependence of the $\mathrm{H}^{-}$detachment cross section on the pulse duration is also examined. We consider single and double coherent pulses, where each pulse comprises in turn a half-cycle, a single-cycle, a few cycles, and several to many cycles. We thus present a comprehensive analysis of results to be expected from short-pulse detachment of negative ions, for which related laser detachment experiments have recently appeared [38]. We expect also that our predictions apply at least qualitatively to ion- ization of neutral atoms with short laser pulses, as suggested by experiments involving Rydberg electron wave packets.

This paper is arranged as follows: In Sec. II we expand the Volkov propagator in terms of Volkov states, and generalize the Keldysh-type formalism for the general case of external fields that may have a nonvanishing vector potential as $t \rightarrow \infty$. We also discuss the validity of this approach for the pulses considered in this paper. We then use our generalized formulation to calculate the detachment rates of $\mathrm{H}^{-}$by HCPs as well as by few-cycle pulses in Sec. III. Our examination of $\mathrm{H}^{-}$detachment by pairs of oppositely directed (i.e., "bidirectional") HCPs allows one to understand the interference pattern seen in detachment by single-cycle pulses. We also examine in detail the transition from few-cycle pulses to many-cycle pulses as the experimental parameters are varied, i.e., the laser frequency and the pulse duration. We then examine the use of pairs of single-cycle pulses, differing in phase by $180^{\circ}$, together with a modest static electric field for coherent control of the extent of $\mathrm{H}^{-}$detachment as a function of the time delay between the pulses. Finally, we examine the use of the absolute phase of the carrier wave with respect to the pulse envelope to control $\mathrm{H}^{-}$detachment. In Sec. IV we summarize our results and draw some conclusions.

\section{THEORY OF $\mathrm{H}^{-}$DETACHMENT BY FEW-CYCLE PULSES}

Photodetachment of $\mathrm{H}^{-}$has been investigated extensively by a variety of theoretical methods. Among these methods are the Keldysh-type formalisms that are well established for treating detachment of negative ions subjected to monochromatic, intense laser fields [15-22]. However, unlike the case of a monochromatic field or even a long laser pulse (which generally contains many cycles of laser oscillation), a halfcycle pulse or a few-cycle pulse may result in a nonvanishing vector potential after it passes the $\mathrm{H}^{-}$ion. When a static electric field is introduced, it may always be represented by a nonvanishing vector potential. The problem of photodetachment of $\mathrm{H}^{-}$in the presence of a static electric field has recently been reviewed by Manakov et al. [39], who present also a detailed analysis of the problem by means of the quasistationary, quasienergy state approach, which takes full account of the electron-atom interactions that are ignored in Keldysh-type approaches. (Such interactions are significant mainly for static fields that are much stronger than typical laboratory static fields, particularly for multiphoton detachment in which an even number of photons are absorbed.) In this paper our aim is to generalize Keldysh-type treatments of the photodetachment of $\mathrm{H}^{-}$for the cases of half- or fewcycle pulses, possibly in the presence of a static electric field. We take a time-dependent approach and examine the time evolution of electron wave packets resulting from the interaction of a pulse with an $\mathrm{H}^{-}$ion. We note that the impulse approximation [9], which is well established for studying the interaction of HCPs with Rydberg atoms, does not apply to the situations considered in this paper because the pulse durations we consider are much longer (by at least one order of 
magnitude) than the Kepler period of the ground-state electron in $\mathrm{H}^{-}$.

In this section, we first derive the Volkov state momentum space solution of the Schrödinger equation for an electron in both a general few-cycle laser pulse and a static electric field. A zero-range potential [40] is used to describe the negative ion $\mathrm{H}^{-}$; it supports a single bound state that has a wellknown wave function. Next, we generalize the Keldysh-type formalism for the case of $\mathrm{H}^{-}$detachment by a general halfcycle or a few-cycle pulse, which may result in a nonzero vector potential at the end of the pulse. We then calculate the probability amplitude for transition from the zero-range potential initial state to a plane-wave final state with welldefined momentum, thereby obtaining the detachment probability density for $\mathrm{H}^{-}$exposed to a few-cycle laser pulse.

\section{A. The analytic Volkov state wave function in momentum space}

Consider a free electron moving in both a few-cycle pulse field and a static electric field. Its motion can be described by the following three-dimensional Schrödinger equation in momentum space (we use atomic units throughout this paper):

$$
i \frac{\partial}{\partial t} \Psi^{(V)}(\mathbf{p}, t)=\left[\frac{1}{2} \mathbf{p}^{2}+\left[E_{s}+E_{P}(t)\right] i \frac{\partial}{\partial p_{z}}\right] \Psi^{(V)}(\mathbf{p}, t) .
$$

Here $\mathbf{p}$ denotes the momentum vector of the detached electron. We assume that both the static electric field $E_{s}$ and the few-cycle pulse field $E_{P}(t)$ are polarized along the $z$ axis, and that the dipole approximation applies. In order to ensure gauge invariance of our results without the necessity for gauge transformation of our wave function [41], we use the length gauge in Eq. (1), in which the coordinate $z$ is represented in momentum space by $i \partial / \partial p_{z}$. Since all fields lie along the $\mathrm{z}$ axis, we may decompose the Volkov state wave function into transverse and parallel components, $\Psi_{\perp}\left(p_{x}, p_{y}, t\right)$ and $\Psi_{\|}\left(p_{z}, t\right)$, where each of these components satisfies its own Schrödinger equation, as follows:

$$
\begin{gathered}
i \frac{\partial}{\partial t} \Psi_{\perp}\left(p_{x}, p_{y}, t\right)=\frac{1}{2}\left(p_{x}^{2}+p_{y}^{2}\right) \Psi_{\perp}\left(p_{x}, p_{y}, t\right), \\
i \frac{\partial}{\partial t} \Psi_{\|}\left(p_{z}, t\right)=\left(\frac{1}{2} p_{z}^{2}++\left[E_{s}+E_{P}(t)\right] i \frac{\partial}{\partial p_{z}}\right) \Psi_{\|}\left(p_{z}, t\right) .
\end{gathered}
$$

Equation (2) has the following simple solution:

$$
\begin{aligned}
\Psi_{\perp, k_{x} k_{y}}\left(p_{x}, p_{y}, t\right)= & \delta\left(k_{x}-p_{x}\right) \delta\left(k_{y}-p_{y}\right) \\
& \times \exp \left[-\frac{i}{2}\left(k_{x}^{2}+k_{y}^{2}\right) t\right],
\end{aligned}
$$

where $k_{x}$ and $k_{y}$ are the transverse momenta of the detached electron. To solve Eq. (3) for the parallel wave function, we first seek its analytic solution in coordinate space, and then we Fourier transform it back to momentum space. In coordinate space, Eq. (3) is

$$
i \frac{\partial}{\partial t} \Psi_{\|}(z, t)=\left(-\frac{1}{2} \frac{\partial^{2}}{\partial z^{2}}+\left[E_{s}+E_{P}(t)\right] z\right) \Psi_{\|}(z, t) .
$$

To solve the above equation, we make the following ansatz:

$$
\begin{aligned}
\Psi_{\|}(z, t) & =\exp \left(-i z \times \int^{t}\left[E_{S}+E_{P}\left(t^{\prime}\right)\right] d t^{\prime}\right) f(z, t) \\
& =e^{i z A(t) / c} f(z, t),
\end{aligned}
$$

where $A(t)=-c \int^{t}\left[E_{s}+E_{P}\left(t^{\prime}\right)\right] d t^{\prime}$ is the vector potential for both the few-cycle pulse field $E_{P}(t)$ and the static electric field $E_{s}$, and $c=137.036$ (in atomic units) is the speed of light in vacuum. Inserting Eq. (6) into Eq. (5), we obtain the following equation for the function $f(z, t)$ :

$$
i \frac{\partial}{\partial t} f(z, t)=\frac{1}{2}\left[-i \frac{\partial}{\partial z}+A(t) / c\right]^{2} f(z, t) .
$$

We see from the form of Eq. (7) that the ansatz in Eq. (6) represents a gauge transformation from the length to the velocity gauge. The solution of Eq. (7) is

$$
f_{k_{z}}(z, t)=(2 \pi)^{-1 / 2} e^{i k_{z} z} \times \exp \left(-\frac{i}{2} \int^{t}\left[k_{z}+A(\tau) / c\right]^{2} d \tau\right) .
$$

We observe that the function $f_{k_{z}}(z, t)$ is characterized by the parallel momentum $k_{z}$ of the detached electron. Finally, we Fourier transform this solution for $\Psi_{\|}(z, t)$ back to momentum space; we obtain

$$
\begin{aligned}
\Psi_{\|, k_{z}}\left(p_{z}, t\right)= & \delta\left(k_{z}+A(t) / c-p_{z}\right) \\
& \times \exp \left(-\frac{i}{2} \int^{t}\left[k_{z}+A(\tau) / c\right]^{2} d \tau\right) .
\end{aligned}
$$

Combining Eqs. (4) and (9), the normalized Volkov (V) wave function in momentum space is

$$
\begin{aligned}
\Psi_{k_{x} k_{y} k_{z}}^{(V)}(\mathbf{p}, t)= & \delta\left(k_{x}-p_{x}\right) \delta\left(k_{y}-p_{y}\right) \delta\left(k_{z}+A(t) / c-p_{z}\right) \\
& \times \exp \left(-i \epsilon_{f} t-i \int^{t}\left[k_{z} A(\tau) / c\right.\right. \\
& \left.\left.+A(\tau)^{2} / 2 c^{2}\right] d \tau\right)
\end{aligned}
$$

where $\epsilon_{f}=\frac{1}{2}\left(k_{x}^{2}+k_{y}^{2}+k_{z}^{2}\right)$ is the kinetic energy of the detached electron.

\section{B. Generalized formalism for $\mathrm{H}^{-}$detachment by few-cycle pulses}

The transition amplitude from an initial state before the laser interaction to a final state $\Psi_{f}(\mathbf{p}, t)$ after the laser interaction is

$$
S_{i \rightarrow f}=\lim _{t \rightarrow \infty}\left\langle\Psi_{f}(t) \mid \Psi(t)\right\rangle,
$$


where the brackets imply integration over the momentum coordinate $\mathbf{p}$. The time-dependent wave function $\Psi(\mathbf{p}, t)$ of the system has developed out of the initial state:

$$
\Psi(\mathbf{p}, t)=\lim _{t^{\prime} \rightarrow-\infty} U\left(\mathbf{p}, t ; \mathbf{p}^{\prime}, t^{\prime}\right) \Psi_{i}\left(\mathbf{p}^{\prime}, t^{\prime}\right),
$$

where $U\left(\mathbf{p}, t ; \mathbf{p}^{\prime}, t^{\prime}\right)$ is the time-evolution operator and $\Psi_{i}\left(\mathbf{p}^{\prime}, t^{\prime}\right)$ is the initial state before the laser interaction. Thus, one may rewrite Eq. (11) as

$$
S_{i \rightarrow f}=\lim _{t \rightarrow \infty, t^{\prime} \rightarrow-\infty}\left\langle\Psi_{f}(t)\left|U\left(t ; t^{\prime}\right)\right| \Psi_{i}\left(t^{\prime}\right)\right\rangle,
$$

where the brackets imply integration over momentum coordinates $\mathbf{p}$ and $\mathbf{p}^{\prime}$. The time-evolution operator $U\left(\mathbf{p}, t ; \mathbf{p}^{\prime}, t^{\prime}\right)$ satisfies the well-known Dyson equation, which in the Schrödinger representation is

$$
\begin{aligned}
U\left(\mathbf{p}, t ; \mathbf{p}^{\prime}, t^{\prime}\right)= & U_{0}\left(\mathbf{p}, t ; \mathbf{p}^{\prime}, t^{\prime}\right)-i \int_{t^{\prime}}^{t} d t^{\prime \prime} \int d^{3} \mathbf{p}^{\prime \prime} \\
& \times U\left(\mathbf{p}, t ; \mathbf{p}^{\prime \prime}, t^{\prime \prime}\right) H_{I}\left(\mathbf{p}^{\prime \prime}, t^{\prime \prime}\right) U_{0}\left(\mathbf{p}^{\prime \prime}, t^{\prime \prime} ; \mathbf{p}^{\prime}, t^{\prime}\right) .
\end{aligned}
$$

In Eq. (14) $U_{0}\left(\mathbf{p}, t ; \mathbf{p}^{\prime}, t^{\prime}\right)$ is the time-evolution operator for the zero-order Hamiltonian, which includes the kineticenergy term and the atomic potential. $H_{I}\left(\mathbf{p}^{\prime \prime}, t^{\prime \prime}\right)$ denotes the interaction Hamiltonian, which includes the laser and static field interactions with the active electron. Substituting the expression for $U\left(\mathbf{p}, t ; \mathbf{p}^{\prime}, t^{\prime}\right)$ into the transition amplitude equation (13) and considering the orthogonality of the final scattering state $\Psi_{f}$ with the initial bound state $\Psi_{i}$, one obtains

$$
S_{i \rightarrow f}=-i \times \lim _{t \rightarrow \infty} \int_{-\infty}^{t} d t^{\prime}\left\langle\Psi_{f}(t)\left|U\left(t ; t^{\prime}\right) \times H_{I}\left(t^{\prime}\right)\right| \Psi_{i}\left(t^{\prime}\right)\right\rangle .
$$

Following the Keldysh-type theory for which the exact time-evolution operator $U\left(p t ; p^{\prime} t^{\prime}\right)$ is approximated by the Volkov propagator $U^{(V)}\left(\mathbf{p}, t ; \mathbf{p}^{\prime}, t^{\prime}\right)$ [15-22] (in which the active electron's interaction with the laser and static fields is treated exactly, but its interaction with the atomic potential is ignored), the transition amplitude from the initial state $\Psi_{i}\left(\mathbf{p}^{\prime}, t^{\prime}\right)$ to a final state $\Psi_{f}(\mathbf{p}, t)$ becomes

$$
S_{i \rightarrow f} \simeq-i \times \lim _{t \rightarrow \infty} \int_{-\infty}^{t} d t^{\prime}\left\langle\Psi_{f}(t)\left|U^{(V)}\left(t ; t^{\prime}\right) \times H_{I}\left(t^{\prime}\right)\right| \Psi_{i}\left(t^{\prime}\right)\right\rangle,
$$

where

$$
H_{I}\left(\mathbf{p}^{\prime}, t^{\prime}\right)=i\left[E_{s}+E_{P}\left(t^{\prime}\right)\right] \partial / \partial p_{z}^{\prime}
$$

is the dipole interaction Hamiltonian in the length gauge. If we choose the Volkov state as the final state, obviously our Eq. (16) reduces to the one used in Ref. [17] (for the case $E_{s}=0$ ) and in Refs. [18,23] (for the case $E_{s} \neq 0$ ). We may expand the Volkov propagator in terms of the Volkov wave functions [42-44],

$$
\begin{aligned}
U^{(V)}\left(\mathbf{p}, t ; \mathbf{p}^{\prime}, t^{\prime}\right)= & -i \theta\left(t-t^{\prime}\right) \int_{-\infty}^{+\infty} \\
& \times d^{3} \mathbf{k} \Psi_{\mathbf{k}}^{(V)}(\mathbf{p}, t) \Psi_{\mathbf{k}}^{(V) *}\left(\mathbf{p}^{\prime}, t^{\prime}\right),
\end{aligned}
$$

where $\theta\left(t-t^{\prime}\right)$ is the Heaviside step function and $\Psi_{\mathbf{k}}^{(V)}(\mathbf{p}, t)$ is given in Eq. (10). Upon substituting Eq. (10) into the above expression, we obtain the Volkov propagator by integrating over k:

$$
\begin{aligned}
& U^{(V)}\left(\mathbf{p}, t ; \mathbf{p}^{\prime}, t^{\prime}\right) \\
& =-i \theta\left(t-t^{\prime}\right) \delta\left(p_{x}-p_{x}^{\prime}\right) \delta\left(p_{y}-p_{y}^{\prime}\right) \\
& \quad \times \delta\left(p_{z}-p_{z}^{\prime}-\left[A(t)-A\left(t^{\prime}\right)\right] / c\right) e^{-(i / 2)\left(p_{x}^{2}+p_{y}^{2}+p_{z}^{2}\right)\left(t-t^{\prime}\right)} \\
& \quad \times \exp \left(-i \int_{t^{\prime}}^{t} d \tau\left\{p_{z}[A(\tau)-A(t)] / c\right.\right. \\
& \left.\left.\quad+[A(\tau)-A(t)]^{2} / 2 c^{2}\right\}\right) .
\end{aligned}
$$

The final state is considered to be a plane wave having momentum $\mathbf{K}$, i.e.,

$$
\Psi_{f, \mathbf{K}}(\mathbf{p}, t)=\delta(\mathbf{K}-\mathbf{p}) e^{-i / 2 \mathbf{K}^{2} t}
$$

We represent the $\mathrm{H}^{-}$ion by the well-known $\delta$ function potential [40], whose bound state is taken to be our initial state. Its wave function $[45,46]$ has the following form in momentum space:

$$
\Psi_{i}\left(\mathbf{p}^{\prime}, t^{\prime}\right)=\frac{B}{\sqrt{2 \pi}} \frac{1}{\mathbf{p}^{\prime 2} / 2-\epsilon_{i}} e^{-i \epsilon_{i} t^{\prime}}
$$

where the normalization parameter is $B=0.31552$ [46] and the ground-state energy is $\epsilon_{i}=-0.027751$ a.u. Physically, Eq. (16) describes a laser and static field-induced transition of an electron initially in the $\mathrm{H}^{-}$bound state to the continuum, where it propagates in the presence of both the laser and the static fields up to time t. At that time the probability amplitude is projected onto a plane-wave state so that its distribution in momentum space can be examined, as in Eq. (26) below.

Substituting the Volkov propagator (19), the final state wave function $\Psi_{f, \mathbf{K}}(20)$, the interaction Hamiltonian $H_{I}$ (17), and the initial state $\Psi_{i}$ (21) into Eq. (16), one obtains the transition amplitude from the initial state to the final state (characterized by the momentum $\mathbf{K}$ ) by integrating over the momenta $\mathbf{p}$ and $\mathbf{p}^{\prime}$,

$$
S_{i \rightarrow f}(\mathbf{K})=\lim _{t \rightarrow+\infty} R_{\mathbf{K}}(t)
$$

where the time-dependent transition amplitude $R_{\mathbf{K}}(t)$ to the momentum state $\mathbf{K}$ is given by 


$$
\begin{aligned}
R_{\mathbf{K}}(t)= & i \frac{B}{\sqrt{2 \pi}} \int_{-\infty}^{t} d t^{\prime} e^{i\left(\epsilon_{f}-\epsilon_{i}\right) t^{\prime}} \exp \left(-i \int_{t^{\prime}}^{t} d t^{\prime \prime}\left\{K _ { z } \left[A\left(t^{\prime \prime}\right)\right.\right.\right. \\
& \left.\left.-A(t)] / c+\left[A\left(t^{\prime \prime}\right)-A(t)\right]^{2} / 2 c^{2}\right\}\right) \\
& \times \frac{\left[E_{s}+E_{P}\left(t^{\prime}\right)\right] \times\left[K_{z}-A(t) / c+A\left(t^{\prime}\right) / c\right]}{\left(\left\{K_{x}^{2}+K_{y}^{2}+\left[K_{z}-A(t) / c+A\left(t^{\prime}\right) / c\right]^{2}\right\} / 2-\epsilon_{i}\right)^{2}},
\end{aligned}
$$

where the detached electron's energy is $\epsilon_{f}=\frac{1}{2}\left(K_{x}^{2}+K_{y}^{2}\right.$ $\left.+K_{z}^{2}\right)$. This is the generalized result for $\mathrm{H}^{-}$detachment for the case of few-cycle pulses (including HCPs), which in general may have a nonvanishing vector potential at the end of the pulse. (If we had assumed that the pulse had a spatial envelope as well as a time envelope, the electron might also "feel" a nonvanishing vector potential if it were to exit the spatial region of the pulse [32].) For long laser pulses, for which the final vector potential is zero, i.e., $A(t \rightarrow \infty)=0$, the above formula for the transition amplitude reduces exactly to the usual Keldysh theory result [15-22]. The transition probability density from the ground state of $\mathrm{H}^{-}$to a particular final state with electron momentum $\mathbf{K}$ is thus

$$
W(\mathbf{K})=\left|S_{i \rightarrow f}(\mathbf{K})\right|^{2},
$$

and the total detachment probability can be obtained by integrating $W(\mathbf{K})$ over all possible electron momenta $\mathbf{K}[47]$,

$$
P=\int_{-\infty}^{+\infty} W(K) d^{3} \mathbf{K}
$$

From the time-dependent transition amplitude $R_{\mathbf{K}}(t)$, one may trace the spatial evolution of a detached electron wave packet. Specifically, the detached electron's probability amplitude in momentum space may be written as

$$
\Psi_{W P}\left(p_{x}, p_{y}, p_{z}, t\right)=\int_{-\infty}^{+\infty} R_{\mathbf{K}}(t) \Psi_{f, \mathbf{K}}\left(p_{x}, p_{y}, p_{z}, t\right) d^{3} \mathbf{K},
$$

where $\Psi_{f, \mathbf{K}}$ is the plane-wave final state (20). This expansion in essence reverses the projection onto plane waves, giving the time-dependent electron probability amplitude in the presence of the external fields in momentum space, i.e.,

$$
\begin{aligned}
& \left|\Psi_{W P}(\mathbf{p}, t)\right|^{2} \\
& =\left|\int_{-\infty}^{t} d t^{\prime} \int d^{3} p^{\prime} U^{(V)}\left(p, t ; p^{\prime}, t^{\prime}\right) H_{I}\left(p^{\prime}, t^{\prime}\right) \Psi_{i}\left(p^{\prime}, t^{\prime}\right)\right|^{2} \\
& =\mid \int_{-\infty}^{+\infty} d^{3} k \Psi_{k}^{(V)}(p, t) \int_{-\infty}^{t} d t^{\prime}\left\langle\Psi_{k}^{(V)}\left(t^{\prime}\right)\left|H_{I}\left(t^{\prime}\right)\right|\right. \\
& \left.\quad \times \Psi_{i}\left(t^{\prime}\right)\right\rangle\left.\right|^{2}
\end{aligned}
$$

Also, from the Fourier transform of this momentum-space probability amplitude, one obtains the spatial behavior of the detached electron's wave packet $[23,24], \phi_{W P}(x, y, z, t)$.

\section{Coherent few-cycle pulses}

In order to study coherent control of $\mathrm{H}^{-}$detachment, we employ coherent superpositions of few-cycle (including halfcycle) pulses. We assume that each few-cycle pulse has a $\sin ^{2}$ temporal shape and is polarized along the $z$ axis. The vector potential for the superposition, $A_{p}(t)$, is defined by

$$
\mathbf{A}_{P}(t)=\left(0,0, A_{P}(t)\right)=\left(0,0,-c \int^{t} E_{P}\left(t^{\prime}\right) d t^{\prime}\right),
$$

where the electric field $E_{P}(t)$ of $N$ coherent few-cycle pulses is expressed as

$$
E_{P}(t)=\sum_{j=0}^{N-1} \begin{cases}E_{j} \times \sin ^{2}\left(\frac{\pi(t-j \tau)}{T_{j}}\right), & 0<t-j \tau \leqslant T_{j} \\ \times \cos \left[\omega_{j}(t-j \tau)+\phi_{j}\right], & \\ 0 & \text { otherwise. }\end{cases}
$$

Here $E_{j}$ denotes the field amplitude of the $j$ th few-cycle pulse, $T_{j}$ stands for its pulse duration, $\tau$ is the time delay between two sequential pulses, and $\phi_{j}$ is the absolute phase of the $j$ th few-cycle pulse with respect to its temporal $\left(\sin ^{2}\right)$ envelope. Note that as $\omega_{j} \rightarrow 0$, the $j$ th pulse becomes a HCP, in which the sign of $E_{j}$ determines the HCP direction. The vector potential in Eq. (28) corresponding to the electric field in Eq. (29) is

$A_{P}(t)$

$$
=\sum_{j=0}^{N-1} \begin{cases}0, & t-j \tau<0 \\ -c E_{j} \times\left[\frac{\sin \left[\omega_{j}(t-j \tau)+\phi_{j}\right]}{2 \omega_{j}}\right. & \\ -\frac{\sin \left[\left(2 \pi / T_{j}+\omega_{j}\right)(t-j \tau)+\phi_{j}\right]}{4\left(2 \pi / T_{j}+\omega_{j}\right)} & \\ \left.-\frac{\sin \left[\left(2 \pi / T_{j}-\omega_{j}\right)(t-j \tau)-\phi_{j}\right]}{4\left(2 \pi / T_{j}-\omega_{j}\right)}\right], & t-j \tau \leqslant T_{j} \\ C(j), & t-j \tau>T_{j}\end{cases}
$$

where the constant $C(j)$ is determined by the continuity of $A_{P}(t)$ at time $t=T_{j}+j \tau$ for $j=N-1$. Examples of halfcycle pulses and few-cycle pulses are shown in Fig. 1, in which the maximum field amplitude shown is $5 \times 10^{6} \mathrm{~V} / \mathrm{cm}$ ( $\sim 0.001$ a.u.). Figures 1(a) and 1(c) present the field amplitude and the vector potential for two half-cycle pulses polarized in opposite directions and separated by a time interval $\tau=100$ fs. Similarly, Figs. 1(b) and 1(d) show two sequential few-cycle $(\simeq$ single cycle) pulses.

The results in Fig. 1 indicate that both the fields and their vector potentials [given by Eqs. (29) and (30)] are continuous, as required. 

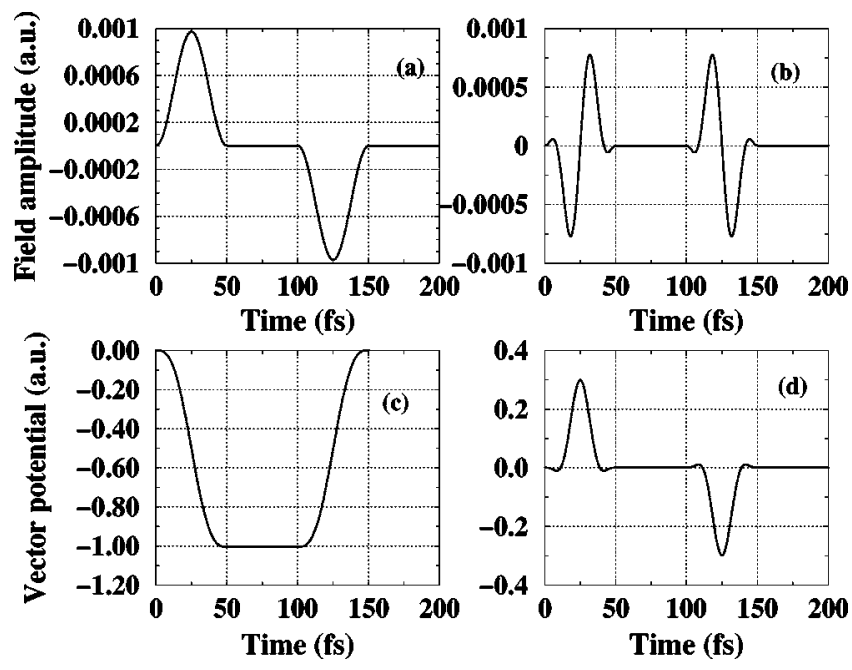

FIG. 1. Examples of the fields considered and their corresponding vector potentials. (a) Field of two half-cycle pulses. (b) Field of two single-cycle pulses ( $\omega=0.0046$ a.u.) (c) Vector potential corresponding to the field in (a). (d) Vector potential corresponding to the field in (b). The maximum field amplitude is $5 \times 10^{6} \mathrm{~V} / \mathrm{cm}$, and each pulse has a duration of $50 \mathrm{fs}$. The time delay $\tau$ between the start of each pulse is $100 \mathrm{fs}$.

\section{Validity of the $S$-matrix approach} and the strong-field approximation

The use of the $S$-matrix approach to describe the interaction of radiation with an atomic system rests, first, on an adiabatically slow turn on of the radiation field at $t=-\infty$ and an adiabatically slow turn off of the field at $t=+\infty$. Second, it assumes implicitly that depletion of the initial state of the atomic system is negligibly small over the course of interaction. For these reasons the use of the $S$-matrix approach to describe the interaction of short laser pulses with an atomic system requires an analysis of whether these conditions apply.

First, regarding adiabaticity, the time of interaction $T$ should be much longer than the atomic orbital period in the system's ground state, i.e.,

$$
T \gg 1 / E_{0} .
$$

Here $T$ is the duration of the pulse and $E_{0}$ is the binding energy of the electron in the initial atomic state. For our few-cycle pulses we have chosen the envelope "frequency" $\omega$ to be approximately $E_{0} / 10$. Hence, for a single-cycle pulse the interaction time is one laser "period," or $T=20 \pi / E_{0}$. Thus, even for a half-cycle pulse Eq. (31) is obviously satisfied.

One may, of course, note that the half-cycle and singlecycle pulses considered in this paper are so short that ascribing a single frequency $\omega$ to them is inappropriate. For this reason we have Fourier analyzed our pulse shapes to obtain the frequency spectrum of our pulses. This analysis indicates that-for all cases considered in this paper-frequency components for which $\omega / E_{0} \geqslant 0.2$ contribute with less than 0.001 times the amplitude of frequencies for which $\omega / E_{0}<0.2$ (i.e., $\left.T>10 \pi / E_{0}\right)$. Thus, the condition for applicability of

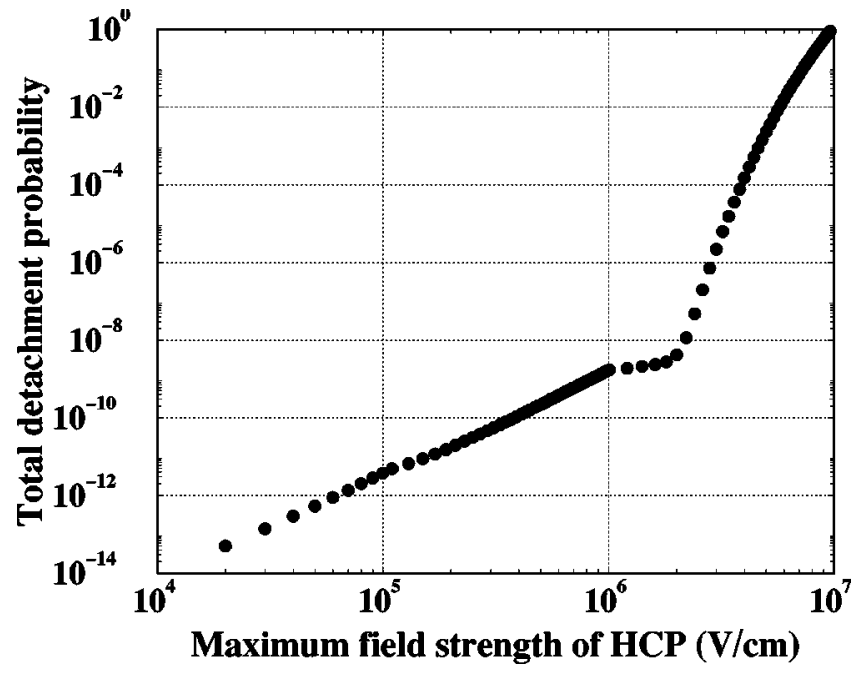

FIG. 2. The detachment probability of $\mathrm{H}^{-}$by a half-cycle pulse (HCP) (with a duration of $100 \mathrm{fs}$ ), as a function of the HCP's maximum field amplitude.

the S-matrix approach given by Eq. (31) holds in all cases considered. We note also that others have used the $S$-matrix approach together with the strong-field approximation to treat few-cycle pulses [25,26].

Another way of looking at the half-cycle pulse case is to consider the limit of a purely static electric field. As already shown in Sec. V C of Ref. [18], the $S$-matrix approach can be used to treat field ionization and the predicted lifetime for the ground state as a function of static electric-field strength agrees well (in the weak field limit) with perturbative predictions of others [48]. In our case, our half-cycle pulses have a peak field amplitude of $5 \times 10^{6} \mathrm{~V} / \mathrm{cm}$. According to Eq. (74) (or Fig. 4) of Ref. [18], a static field of this strength would give the $\mathrm{H}^{-}$ion ground state a lifetime of $0.59 \times 10^{-11} \mathrm{~s}$, or a field ionization rate of $1.7 \times 10^{11} / \mathrm{s}$, If we approximate our 50 -fs long half-cycle pulse by a 50 -fs square pulse with amplitude $5 \times 10^{6} \mathrm{~V} / \mathrm{cm}$, then the probability of ionization is $50 \mathrm{fs} \times 1.7 \times 10^{11} / \mathrm{s}=8.5 \times 10^{-3}$. Thus depletion of the $\mathrm{H}^{-}$ ground state is $\leqslant 0.85 \%$, which again points to the validity of the $S$-matrix approach for the kinds of pulses considered here.

To check further the validity of our generalized Keldyshtype formalism, we consider the detachment probability of $\mathrm{H}^{-}$vs the maximum field amplitude of one half-cycle pulse. The result obtained using Eq. (25) and a HCP duration of 100 fs is shown in Fig. 2. As Fig. 2 indicates, the probabilities for maximum field strengths less than $10^{6} \mathrm{~V} / \mathrm{cm}$ are very small and have an approximately linear increase in the logscale plot, which is typical of the perturbation regime. The detachment probability rises sharply above a field strength of $2 \times 10^{6} \mathrm{~V} / \mathrm{cm}$. This agrees very well with the experimental and theoretical critical field strength [of $(2-3)$ $\left.\times 10^{6} \mathrm{~V} / \mathrm{cm}\right]$ for $\mathrm{H}^{-}$detachment by static electric fields $[48,49]$. A similar "threshold" behavior for the field dependence of the $\mathrm{H}^{-}$detachment probability has been predicted using the quasistationary, quasienergy state approach [50]. Note finally that in the high static field regime, the field dependence of the detachment probability shown in Fig. 2 is 
consistent with predictions of the ionization probability given by the Ammosov, Delone, and Krainov theory for the tunneling rate [51].

As is usual in Keldysh-type theories, we have made the strong-field approximation [i.e., we have replaced the exact time propagator $U\left(p t ; p^{\prime} t^{\prime}\right)$ by the Volkov time-evolution propagator $\left.U^{(V)}\left(p t ; p^{\prime} t^{\prime}\right)\right]$ in deriving our generalized formalism for $\mathrm{H}^{-}$detachment by HCPs or by few-cycle pulses. For this reason, we have chosen large maximum field strengths for the HCPs or few-cycle pulses (so that the interactions lie in the tunneling regime). Specifically, the field amplitude of all HCPs and few-cycle pulses considered in this paper is $5 \times 10^{6} \mathrm{~V} / \mathrm{cm}$.

\section{RESULTS AND DISCUSSION}

Using the generalized Keldysh-type formalism presented above, we examine here the detachment of $\mathrm{H}^{-}$using halfcycle, single-cycle, and few-cycle pulses. Our emphasis is on the coherent control of these processes. First, we examine the use of two HCPs in the cases in which their electric fields are in the same direction or in opposite directions (bidirectional). For the case of bidirectional double HCPs, we observe quantum interference in the detached electron spectra, with the time delay between the bidirectional double HCPs serving as a sensitive control parameter. Second, we investigate the effect of the pulse duration and laser frequency on the $\mathrm{H}^{-}$ detachment process, i.e., we examine the transition from few- to many-cycle pulses. Third, we examine the use of coherent pairs of single-cycle pulses to detach $\mathrm{H}^{-}$in the presence of a static electric field. Our numerical results show that modulations of the total detachment probability of $\sim 30 \%$ can be obtained. Finally, we investigate the use of the absolute phase of single-cycle pulses to control $\mathrm{H}^{-}$detachment.

\section{A. $\mathrm{H}^{-}$detachment by HCPs}

We analyze in this subsection the HCP detachment of $\mathrm{H}^{-}$ in detail. If in Eq. (29) we set $\omega_{j}=0$ and $\phi_{j}=0$, we obtain a $\sin ^{2}$ half-cycle pulse of duration $T_{j}$. Pulses with this shape have been used extensively to describe experimentally produced half-cycle pulses [13].

For a single $\mathrm{HCP}$ with a duration of $50 \mathrm{fs}$, the $\mathrm{H}^{-}$detachment probability density as a function of the electron momenta $K_{x}$ and $K_{z}$ is drawn in Fig. 3(a). The maximum transition probability is located at $K_{x}=0, \quad K_{y}=0, \quad K_{z}$ $\simeq-0.5$ a.u. in momentum space. This numerical result indicates that the most probable tunneling occurs at the peak field of the half-cycle pulse, following which the electron that tunnels out (with zero initial velocity) is accelerated by the residual half part of the $\mathrm{HCP}$, thereby resulting in a final momentum equal to half the vector potential, i.e., $K_{z}=A / 2$. (Note that the vector potential $A$ is $A \simeq-1.0$ a.u. for the HCP considered here.) This situation is very different from the case of using a HCP to impart a kick to Rydberg electrons, following which the final electron momentum is $K_{f}$ $=K_{i}+A$, according to the impulse approximation [7].

Figure 3(b) shows the time evolution of the detached wave packet,

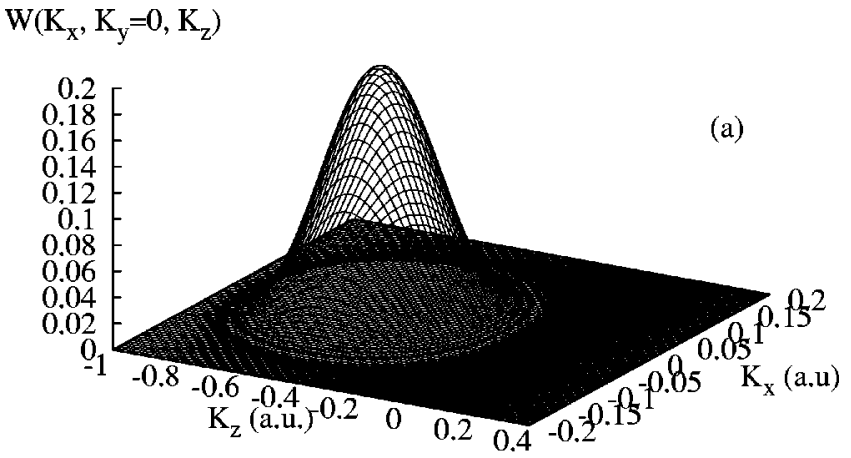

$P(z, t)$

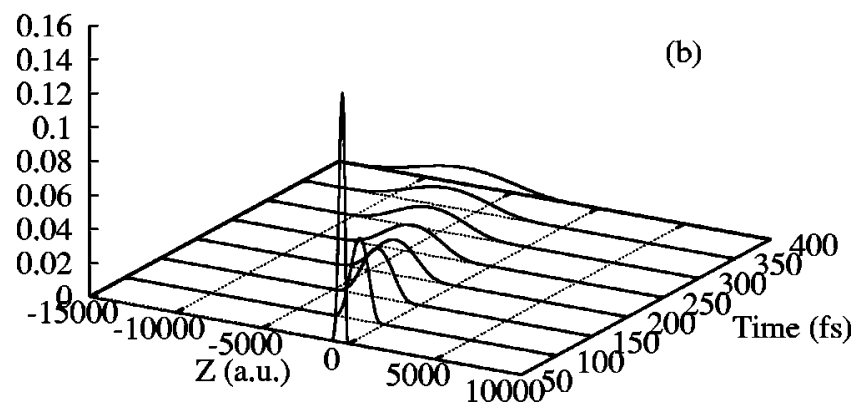

FIG. 3. (a) The transition probability density $W\left(K_{x}, K_{y}=0, K_{z}\right)$ [see Eq. (24)] for detachment of $\mathrm{H}^{-}$by a single half-cycle pulse (HCP), as a function of the final momenta $K_{x}$ and $K_{z}$. Note that the 50 -fs HCP is polarized along the positive $z$ axis, with a maximum field strength of $5 \times 10^{6} \mathrm{~V} / \mathrm{cm}$. (b) The spatial electron wave packet, $P(z, t)=\iint\left|\phi_{W P}(x, y, z, t)\right|^{2} d x d y$, plotted vs $z$ and $t$.

$$
P(z, t)=\iint\left|\phi_{W P}(x, y, z, t)\right|^{2} d x d y,
$$

along the polarization direction (i.e., the $z$ axis) in coordinate space. We see that just after the interaction of the 50-fs HCP, a very narrow wave packet appears, which then moves toward the negative $z$ direction (the field is pointing along the positive $z$ axis). One sees also the large spread of the wave packet for large times.

Figure 4 shows results for the case of two half-cycle pulses pointing in the same direction $(+z)$, with the $\mathrm{H}^{-}$ detachment probability density plotted as a function of the momenta $K_{x}$ and $K_{z}$ of the detached electron. The second

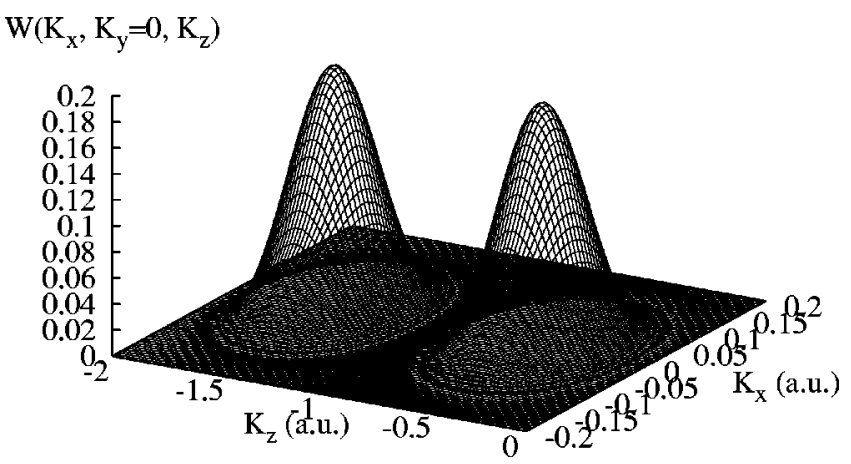

FIG. 4. Similar to Fig. 3(a) but for double HCPs, which are both polarized along the positive $z$ axis. 


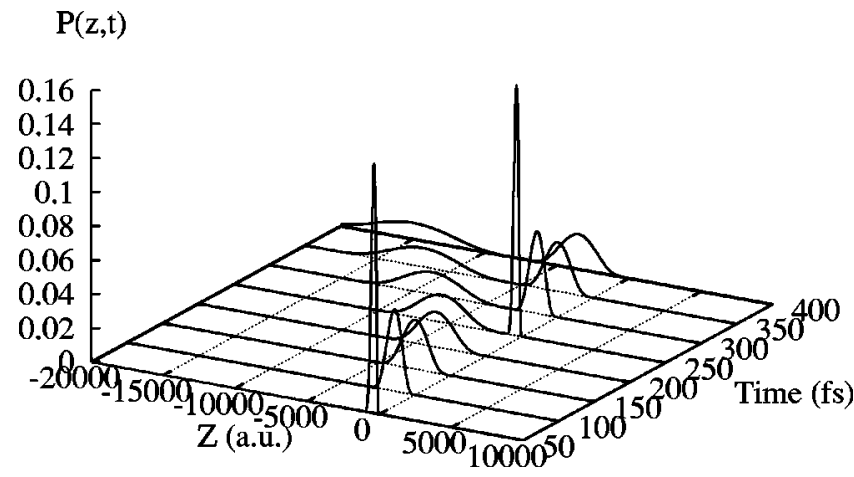

FIG. 5. The spatial wave packet $P(z, t)$ plotted vs $z$ and $t$ for the case of two HCPs polarized in the same direction (along the positive $z$ axis).

HCP is delayed by 200 fs from the first one. In this case, there are two peaks appearing in the continuum. One is located at $K_{z}=\frac{3}{2} A$ and the other is at $K_{z}=\frac{1}{2} A$. These momentum values indicate that the first wave packet receives the full impulse of the second half-cycle pulse, increasing its momentum from $K_{z} \sim \frac{1}{2} A$ to $K_{z} \sim \frac{3}{2} A$, while the second wave packet, resulting from ionization at the peak of the second half-cycle pulse, receives an impulse from the last half of the second pulse equal to $K_{z} \sim \frac{1}{2} A$.

Figure 5 shows the time evolution of the corresponding electron wave packets. Since both HCPs are pointing in the same direction, the first HCP-produced wave packet evolves freely for $200 \mathrm{fs}$, whereupon it is kicked by the second HCP. Note that the numerically calculated impulse of the second $\mathrm{HCP}$ on the initially produced wave packet is just equal to the vector potential of the second $\operatorname{HCP}(A \simeq-1.0$ a.u. $)$. Not only does the second HCP kick the already-produced wave packet, it also drives an additional portion of the still bound probability amplitude to tunnel through the barrier. Thus, one sees eventually two wave packets moving with different velocities but in the same direction.

We now consider a more interesting situation: two halfcycle pulses pointing in opposite directions; in some references they are called bidirectional HCPs [52]. Figure 6 shows the $\mathrm{H}^{-}$detachment probability density $W\left(K_{x}=0, K_{y}\right.$ $=0, K_{z}$ ) vs the electron momentum $K_{z}$ for the following cases: (a) only one HCP pointing in the $+z$ direction; (b) only one HCP pointing in the $-z$ direction; (c) both HCPs acting sequentially with a time delay of $200 \mathrm{fs}$. The pulse parameters are the same as those above, i.e., all HCPs have a maximum field amplitude of $5 \times 10^{6} \mathrm{~V} / \mathrm{cm}$ and a duration of 50 fs.

The most interesting feature of the double HCP case in which the two pulses point in opposite directions is the appearance of quantum interference, as shown in Fig. 6(c). The interference pattern shows that for values of $K_{z}$ in the vicinity of 0.5 a.u., the transition probability is enhanced by a factor of 4, while for other values of $K_{z}$ the transition probability decreases to near zero. One can understand the interference process as follows: the first HCP produces a wave packet that moves freely away from the origin. After a time delay, the second HCP (with opposite field direction) interacts with the entire system (including both the already out-

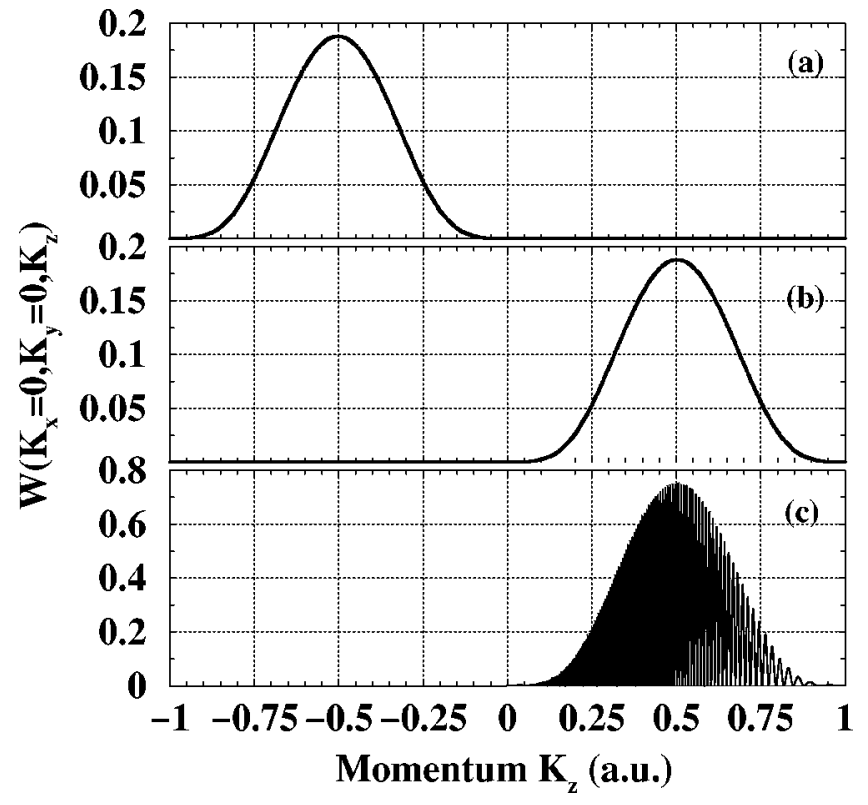

FIG. 6. The detachment probability density $W\left(K_{x}=0, K_{y}\right.$ $=0, K_{z}$ ) plotted as a function of the electron momentum $K_{z}$. (a) Result for only one HCP polarized along the positive $z$ axis; (b) result for one HCP polarized along the negative $z$ axis; (c) result for both HCPs in (a) and (b) in sequence, in which the second one is delayed by $200 \mathrm{fs}$. Both HCPs have a maximum field amplitude of $5 \times 10^{6} \mathrm{~V} / \mathrm{cm}$ and a duration of $50 \mathrm{fs}$.

going wave packet and the $\mathrm{H}^{-}$bound state). The second HCP's action not only makes the bound-state tunnel through the barrier, thereby forming a new wave packet, but also drives the first wave packet back to interfere with the newly produced one. This is clearly seen from the spatial wave packet's temporal evolution shown in Fig. 7.

Our results in Figs. 6(c) and 7 may be interpreted analytically using our general result in Eq. (23) for the transition amplitude. Specifically, consider the bidirectional double HCP shown in Fig. 1(a). Using the analytic expressions in Eqs. (29) and (30) for the electric field $E(t)$ and vector potential $A(t)$ that are shown respectively in Figs. 1(a) and 1(c) (and which are both polarized along the $z$ axis), we may obtain by direct substitution into Eq. (23) the transition amplitude resulting from the bidirectional double HCP (which

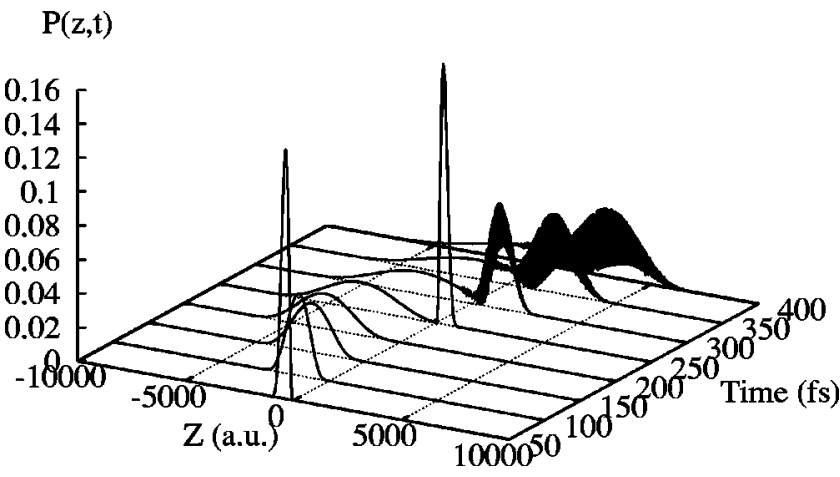

FIG. 7. The time evolution of the electron wave packet $P(z, t)$ along the $z$ axis for the double HCP case of Fig. 6(c). 
ionizes an electron to a free particle state with momentum $\mathbf{K}$ at the time $t=\tau+T$, where $T$ is the duration of each of the HCPs and $\tau$ is the time interval between the starting times of each of the two pulses). The result may be expressed as follows:

$$
\begin{aligned}
R_{\mathbf{K}}^{(1+2)}(t \geqslant \tau+T)= & \left\{\int_{-\infty}^{0} d t^{\prime}+\int_{0}^{T} d t^{\prime}+\int_{T}^{\tau} d t^{\prime}+\int_{\tau}^{\tau+T} d t^{\prime}\right\} \\
& \times e^{i\left(\epsilon_{f}-\epsilon_{i}\right) t^{\prime}} \times \exp \left\{-i \int_{t^{\prime}}^{t} d t^{\prime \prime}\left[K_{z} A\left(t^{\prime \prime}\right)\right.\right. \\
& \left.\left.+A^{2}\left(t^{\prime \prime}\right) / 2\right]\right\} \times F\left(\mathbf{K}, t^{\prime}\right)
\end{aligned}
$$

where

$$
F\left(\mathbf{K}, t^{\prime}\right)=i \frac{B}{\sqrt{2 \pi}} \frac{E\left(t^{\prime}\right)\left[K_{z}+A\left(t^{\prime}\right)\right]}{\left(\left\{K_{x}^{2}+K_{y}^{2}+\left[K_{z}+A\left(t^{\prime}\right)\right]^{2}\right\} / 2-\epsilon_{i}\right)^{2}}
$$

and the superscript " $1+2$ " on the rate in Eq. (33) indicates that this is the total rate for the two bidirectional HCPs.

Owing to the fact that the electric field $E\left(t^{\prime}\right)$ in Eq. (34) is zero except during the time intervals $0<t^{\prime}<T$ and $\tau<t^{\prime}$ $<\tau+T$, only the second and fourth integrals over $t^{\prime}$ in Eq. (33) contribute. Thus the general result for the transitionprobability amplitude may be expressed in terms of two integrals, each over the time period of one of the two bidirectional HCPs. An interesting question is how this general result for the bidirectional case may be expressed in terms of the results for each of the two HCPs acting separately. The result (obtained by straightforward but lengthy algebra) is given in Eq. (35); it is obtained by expressing Eq. (33) in terms of the separate results for each of the two HCPs acting alone:

$$
\begin{aligned}
R_{\mathbf{K}}^{(1+2)}(t=\tau+T)= & R_{\mathbf{K}+\mathbf{A}(T)}^{(1)}(T) \times \exp \left[i f\left(K_{z}, t=\tau+T, \tau\right)\right] \\
& +R_{\mathbf{K}}^{(2)}(t=\tau+T)
\end{aligned}
$$

where

$$
\begin{aligned}
f\left(K_{z}, t=\tau+T, \tau\right)= & \frac{1}{2} K_{z}^{2}(\tau+T)-\frac{1}{2}\left[K_{z}+A(T)\right]^{2} \tau \\
& -\frac{1}{2} \int_{\tau}^{\tau+T}\left[K_{z}+A\left(t^{\prime}\right)\right]^{2} d t^{\prime},
\end{aligned}
$$

and $R_{\mathbf{K}+A(T)}^{(1)}(T)$ is obtained from Eq. (23) for the case of the first HCP shown in Figs. 1(a) and $1(\mathrm{c})$ and $R_{\mathrm{K}}^{(2)}(\tau+T)$ is obtained from Eq. (23) for the case of the second HCP shown in these figures. Note that the first pulse ends at $t$ $=T$, at which time the electron has a mechanical momentum of $\mathbf{K}+A(T)$. [After the second pulse, which adds an impulse $-A(T)$, the momentum of the first wave packet will be the same as that of the second wave packet.] The transitionprobability amplitude $R_{\mathbf{K}+\mathbf{A}(T)}^{(1)}$ is multiplied by a phase factor that comprises three terms [cf. Eq. (36)] whose origins

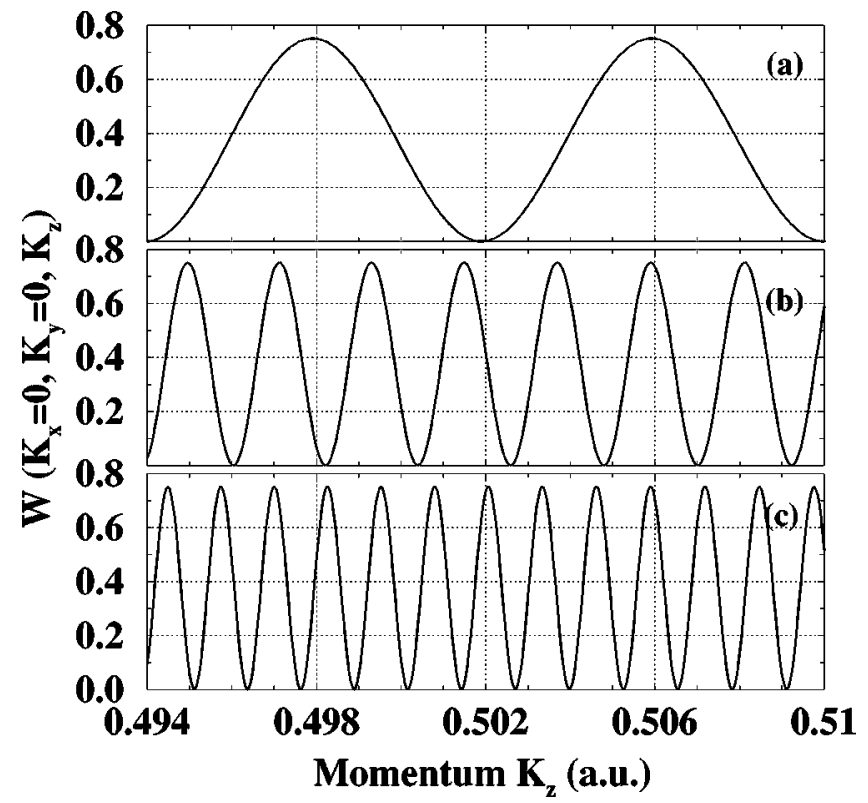

FIG. 8. Transition-probability density for $\mathrm{H}^{-}$by double HCPs for different time delays: (a) $50 \mathrm{fs}$, (b) $150 \mathrm{fs}$, and (c) $250 \mathrm{fs}$.

may be interpreted simply as follows: (1) Free evolution with kinetic energy $\frac{1}{2} K_{z}^{2}$ for a time $\tau+T$ (the duration of the bidirectional pair of HCPs); (2) free evolution with kinetic energy $\frac{1}{2}\left[K_{z}+A(T)\right]^{2}$, the kinetic energy of the electron after the first HCP, for a time $\tau$; and (3) the phase accumulation during the action of the second HCP. Taking the absolute square of Eq. (35) we obtain the transition-probability density, in which interference terms appear. Thus, the total transition-probability density oscillates as the momentum $K_{z}$ of the detached electron varies, as may be seen in Figs. 6(c) and 7. [Note that the oscillations shown in Fig. 6(c) as $K_{z}$ increases depend not only on the phase function $f\left(K_{z}, \tau\right.$ $+T, \tau)$ but also on the relative phases of $R_{\mathbf{K}+A(T)}^{(1)}$ and $R_{\mathbf{K}}^{(2)}$.] The phase between the two overlapping wave packets depends on the time delay $\tau$ as well. Thus, one can control the detached electron spectra by adjusting $\tau$. This dependence on $\tau$ arises only from the phase function $f\left(K_{z}, \tau+T, \tau\right)$ in Eq. (36). As an example, Fig. 8 shows the electron spectra as a function of the electron momentum $K_{z}$ for the same double bidirectional HCPs for different time delays: (a) $\tau=50 \mathrm{fs}$, (b) $\tau=150 \mathrm{fs}$, and (c) $\tau=250 \mathrm{fs}$. The longer the time delay between two HCPs, the more dense the interference pattern is as a function of $K_{z}$. This behavior is in accord with Eq. (36). Finally, note that although the maximum probability density in Fig. 6(c) is four times greater than that of either of the two pulses in Fig. 6(a) or 6(b), the integrated probability (over $K_{z}$ ) equals the sum of the integrated probabilities of the two separate pulses.

\section{B. Dependence of the $\mathrm{H}^{-}$detachment probability on the number of cycles in a laser pulse}

In the above section we discussed the process of $\mathrm{H}^{-}$detachment for the case of half-cycle pulses that can be taken as the pulse envelope for a field with frequency $\omega=0$. Al- 

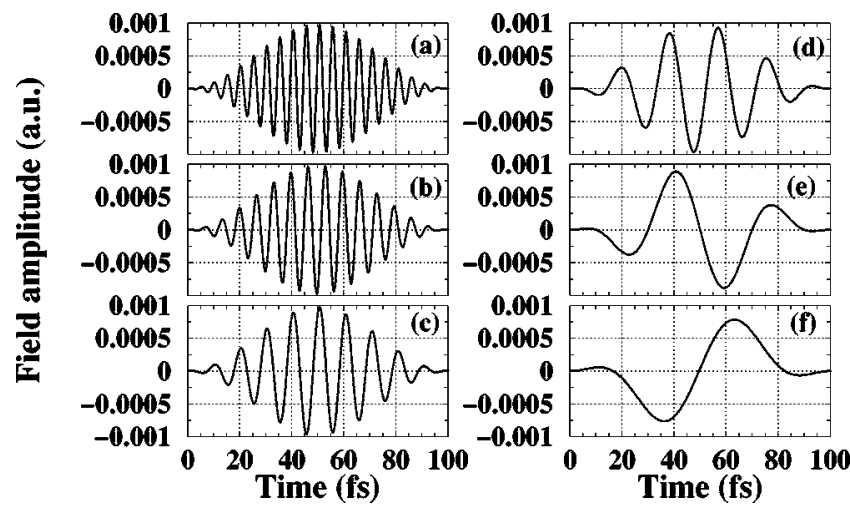

FIG. 9. Few-cycle laser pulse fields with the same duration (100 fs), but different frequencies: (a) 0.03 a.u., (b) 0.023 a.u., (c) 0.015 a.u., (d) 0.008 a.u., (e) 0.0038 a.u., (f) 0.0023 a.u.

though the laser frequency dependence of $\mathrm{H}^{-}$detachment has been investigated for long (many-cycle) pulses, we investigate in this section the variation of $\mathrm{H}^{-}$detachment as the number of cycles in a laser pulse varies from very few to very many. We investigate first the frequency variation of $\mathrm{H}^{-}$ detachment for a laser pulse of fixed duration. We then examine the dependence of $\mathrm{H}^{-}$detachment on laser pulse duration for the case of a fixed laser frequency.

\section{Frequency dependence of $\mathrm{H}^{-}$detachment for a laser pulse of fixed duration}

We consider here the case of pulses having the same duration, but for which the laser frequency varies and is small but nonzero. That is, we investigate the case of few-cycle laser pulses, which provides dramatically different results for $\mathrm{H}^{-}$detachment from that of the half-cycle pulse case. The laser fields considered are shown in Fig. 9, where in the panels (a)-(f) the laser frequency decreases from $\omega$ $=0.03$ a.u. to 0.0023 a.u.

All of the laser pulses have a maximum field amplitude of $5 \times 10^{6} \mathrm{~V} / \mathrm{cm}$, with the same duration of $100 \mathrm{fs}$. For each of these pulses, the corresponding $\mathrm{H}^{-}$detachment probability densities are shown as a function of the detached electron momentum $K_{z}$ in Fig. 10. Since the binding energy of $\mathrm{H}^{-}$is $\epsilon_{i}=-0.027751$ a.u., one-photon detachment should occur for the field shown by Fig. 9(a) ( $\omega=0.03$ a.u.). This is shown in Fig. 10(a) in which the detached electron momentum is given by the energy conservation requirement [i.e., $K_{z}= \pm \sqrt{2\left(n \hbar \omega-\left|\epsilon_{i}\right|\right)}$ with $\left.n=1\right]$. Figure 10(b) shows explicitly the two-photon detachment process. In Fig. 10(c) the two-photon process is near the detachment threshold, so the $\pm K_{z}$ peaks come together near zero. Three-photon peaks at $K_{z}= \pm 0.2848$ a.u. and $K_{z}= \pm 0.18$ a.u. are also visible in Figs. 10(b) and 10(c), respectively. All these peaks are exactly located at the positions that are expected from the energy conservation requirement.

When the laser frequency decreases further, the 100-fs pulse has fewer and fewer oscillations [see Figs. 9(d)-9(f)]. The corresponding $\mathrm{H}^{-}$detachment probability density decreases, and instead of double peaks one finds in Figs. 10(d)-10(f) many peaks. A very interesting feature in Fig.
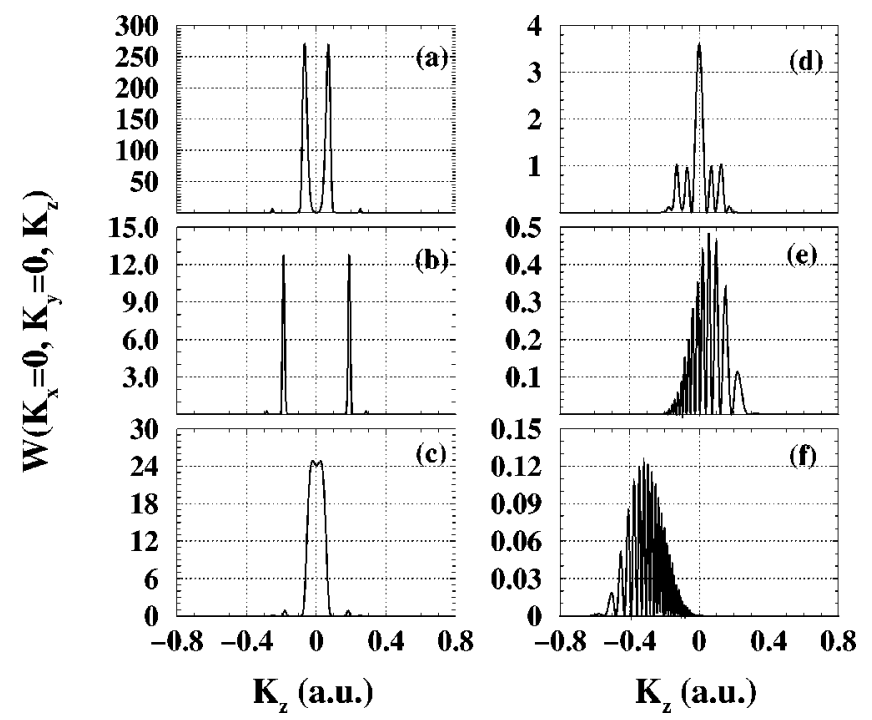

FIG. 10. Detachment probability for $\mathrm{H}^{-}$using the fields shown in the corresponding panels of Fig. 9.

10(f) is that all the detached electrons move in the same direction. This contrasts with the case of many-cycle pulses, as seen in Figs. 10(a)-10(c), in which the electron wave packet absorbs one or two photons, and then moves along both the positive and the negative directions along the laser polarization axis with equal probability.

Rather the behavior observed in Fig. 10(f) for an essentially single-cycle pulse [cf. Fig. 9(f)] is consistent with the case of using bidirectional HCPs [Fig. 6(c)]. In fact, the almost one-cycle field shown in Fig. 9(f) can be approximated by two half-cycle pulses, of which the first is a negative HCP while the second is a positive HCP. As plotted in Fig. 6(c), the detached electron wave packet is ejected predominantly in one direction. This feature of few-cycle pulses opens the possibility of coherent control of $\mathrm{H}^{-}$detachment by employing coherent few-cycle pulses and an external static electric field, as we show in Sec. III C.

\section{Pulse duration dependence of $\mathrm{H}^{-}$detachment for fixed laser frequency}

We investigate here the pulse duration dependence of $\mathrm{H}^{-}$ detachment for the case of pulses having $\simeq 3-15$ cycles per pulse, with each pulse having the same maximum amplitude. We choose a laser frequency of $\omega=0.022$ a.u., for which the single-photon detachment channel is closed for long pulses since the electron affinity of $\mathrm{H}^{-}$is $I_{p}=0.027751$ a.u. For a long pulse, therefore, the only possible detachment channels are those involving absorption of two or more photons. Figure 11(a) shows the $\mathrm{H}^{-}$detachment probability density $W\left(K_{x}, K_{y}=0, K_{z}\right)$ for a $\sin ^{2}$ laser pulse of duration $T$ $=100 \mathrm{fs}$ (about 15 cycles). [Note that $K_{y}=0$ is a special case of the general probability density $W\left(K_{x}, K_{y}, K_{z}\right)$; the kinetic energy of the detached electron is $E_{K}=\mathbf{K}^{2} / 2$.] One sees clearly the two-photon detachment peaks that are located on the "energy conservation circle" defined by $\frac{1}{2}\left(K_{x}^{2}\right.$ $\left.+K_{z}^{2}\right)=2 \omega-I_{p}$. As expected, there are no peaks inside the energy conservation circle for two-photon detachment. The 


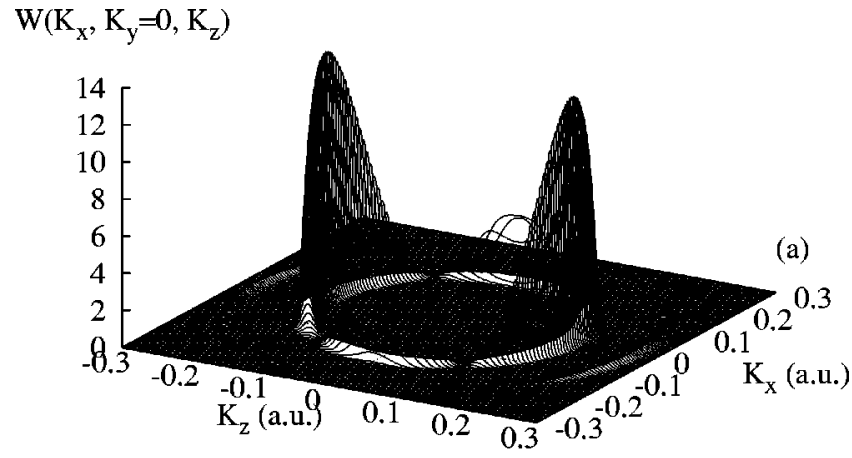

$\mathrm{W}\left(\mathrm{K}_{\mathrm{x}}, \mathrm{K}_{\mathrm{y}}=0, \mathrm{~K}_{\mathrm{z}}\right)$

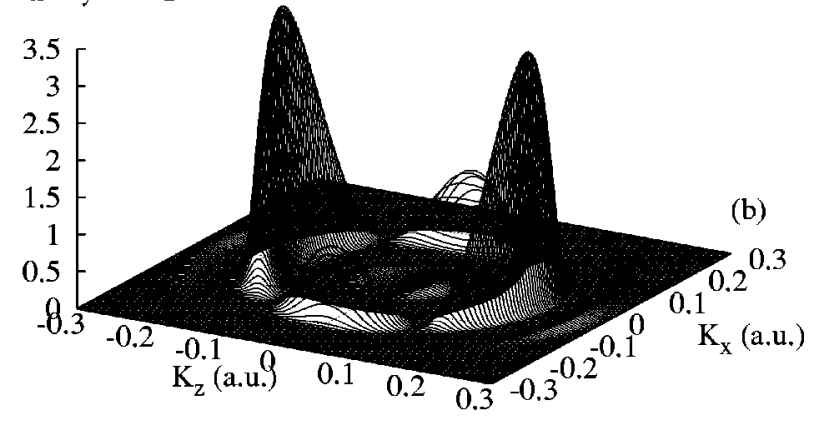

$\mathrm{W}\left(\mathrm{K}_{\mathrm{x}}, \mathrm{K}_{\mathrm{y}}=0, \mathrm{~K}_{\mathrm{z}}\right)$

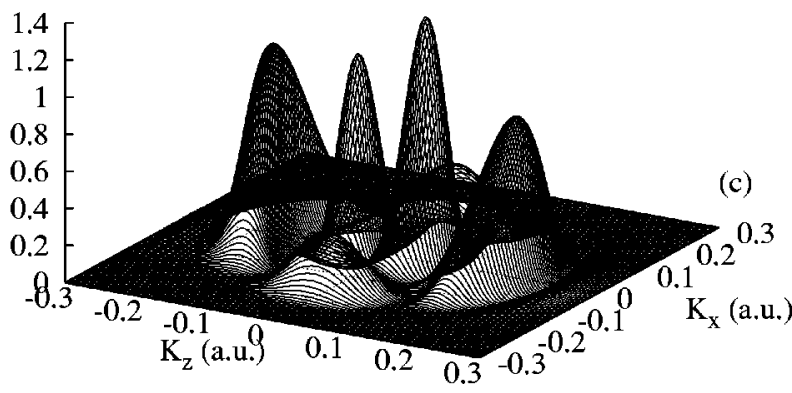

FIG. 11. The detachment of $\mathrm{H}^{-}$by ultrashort laser pulses, with different pulse durations: (a) $100 \mathrm{fs}$, (b) $50 \mathrm{fs}$, and (c) $25 \mathrm{fs}$. The laser central frequency is the same for all three cases: $\omega$ $=0.022$ a.u.

dominant peaks for two-photon detachment lie along the laser polarization direction (i.e., the $z$ axis, for which $K_{x}=0$ ). However there exist also two other small peaks appearing perpendicular to the $z$ axis (i.e., having $K_{z}=0$ ). Notice also that on the energy conservation circle there exist some very deep "valleys" in which the detachment probability density is close to zero. The peaks perpendicular to the laser polarization axis as well as the minima along the energy conservation circle may be understood as arising from interference between $s$-wave and $d$-wave final-state electron channels populated by two-photon transitions [53,54]. Note finally that the energy conservation circle for three-photon detachment is also visible in Fig. 11(a).

For shorter pulse envelope durations it is necessary to take into account the fact that our pulse is of finite duration. For $T=100$-fs pulses, as shown in Fig. 11(a), this was unnecessary: as a Fourier analysis of our finite pulse shows, for $T=100 \mathrm{fs}$, frequency components differing from the carrier frequency by more than $10 \%$ or so have amplitudes at least four orders of magnitude smaller. For shorter pulse envelopes, however, the width of the carrier frequency component increases and the amplitudes of other frequency components increase. While these higher frequency components are still very weak compared to the carrier frequency component, they may not be weak compared to the amplitude for a two-photon process at the carrier frequency.

In Fig. 11(b) we show results for a pulse envelope of $T$ $=50 \mathrm{fs}$. For this case the carrier frequency component of the laser pulse is broadened, but not so much as to overlap the one-photon threshold. Therefore the main process observed is still two-photon detachment, as in Fig. 11(a). Because the laser pulse is shorter by a factor of 2 from that in Fig. 11(a), the amplitude of the two-photon detachment probability density is smaller by roughly a factor of 4 . All features of the two-photon energy conservation circle are present, as discussed above for Fig. 11(a); the three-photon detachment process is also observed. What is new here is a small ring of detachment probability inside the two-photon energy conservation circle. Our Fourier analysis of our pulse identifies this innermost ring as due to a frequency component that peaks at a frequency of $\omega=0.0292$ a.u., which is just above the onephoton detachment threshold at 0.027751 a.u. Although the amplitude of this component of our pulse is nearly four orders of magnitude smaller than that of our carrier wave frequency component, the carrier wave component is below the one-photon detachment threshold. The innermost ring is therefore only visible because it is producing one-photon detachment and is being compared to the two-photon process produced by the carrier wave frequency component.

In Fig. 11(c) we show results for an even shorter pulse envelope having $T=25 \mathrm{fs}$. In this case a Fourier analysis of our pulse shows that the carrier wave frequency component of the laser pulse is so broad that the high-energy portion of the carrier wave frequency component lies above the onephoton threshold. This greater width of the carrier wave frequency component is exhibited in the greater width of the peaks along the two-photon energy conservation circle. Now, however, there exist two twin peaks in the vicinity of $K_{z}$ $=0$ (i.e., along the laser polarization axis) that arise from one-photon detachment. Because of the Wigner threshold law, the detachment probability density is precisely zero at the one-photon threshold, but rises above threshold. As the central frequency component amplitude drops sharply with energy above threshold, these peaks are well localized (at $K_{z} \simeq \pm 0.0488$ a.u.), as shown in Fig. 11(c). Note finally that here we have a situation in which the amplitude of the onephoton detachment process is comparable to that of the twophoton process.

\section{Coherent control of $\mathbf{H}^{-}$detachment using single-cycle pulses and a static electric field}

The coherent control scheme we investigate here consists in using a static electric field to reflect a detached electron wave packet back to the origin, whereupon a second pulse is used to produce a second electron wave packet (coherent with the first) that overlaps and interferes with the reflected 


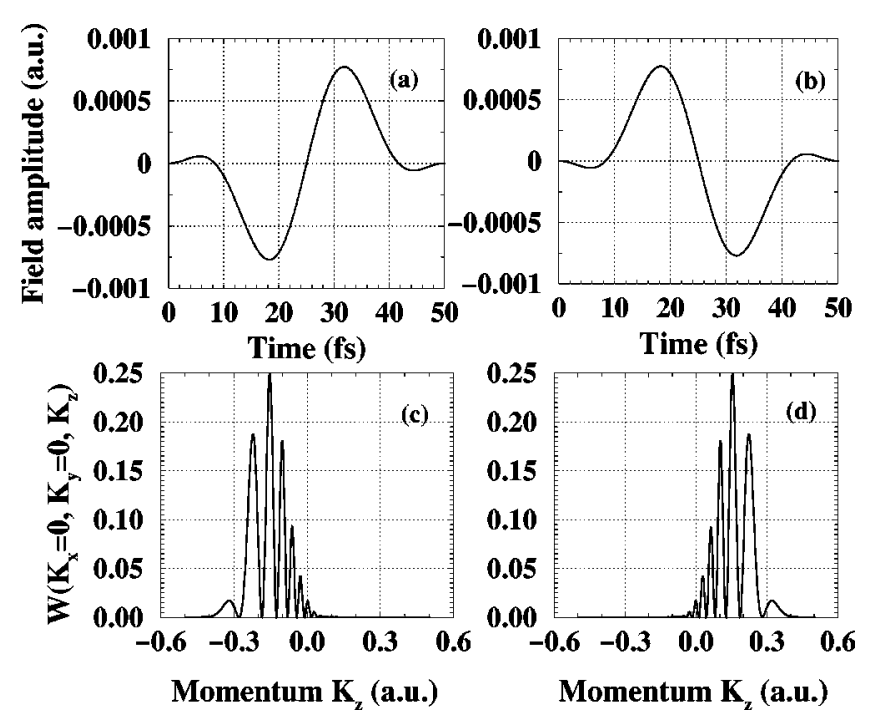

FIG. 12. The $\mathrm{H}^{-}$detachment probability density $W\left(K_{x}=K_{y}\right.$ $=0, K_{z}$ ) [cf. Eq. (24)] along the $z$ axis for the case of single-cycle pulses. (a) and (b) indicate the two single-cycle pulses, which are phase shifted by $\pi$ with respect to each other. Both pulses have the same frequency ( $\omega \simeq 0.0046$ a.u.) and the same duration (50 fs). (c) and (d) show the transition-probability density as a function of electron momentum $K_{z}$, corresponding, respectively, to fields (a) and (b).

one. This scheme has been investigated for the case of manycycle, short laser pulses both for $\mathrm{H}^{-}$detachment $[23,24]$ and for highly excited Rydberg state wave packets [36,37]. As we have discussed above, the outgoing electron wave packets generated by a many-cycle laser pulse move outward in both directions along the laser polarization axis. So, in principle, only $50 \%$ of outgoing wave packets can be reflected back to the origin by a static electric field. With this scheme the maximum modulation of the total detachment cross section depends also on how much time it takes for an electron wave packet to reach the static electric-field potential barrier, be reflected, and then return to the origin. The longer this excursion takes, the greater the spread of the wave packet in the direction perpendicular to the laser polarization axis, and hence the smaller the overlap with the electron wave packet produced by a second pulse from the ground state. For the laser pulse and static field parameters employed in Refs. [23] and [24] modulations of the $\mathrm{H}^{-}$detachment cross section of $2 \%$ and $10 \%$, respectively, were predicted. In contrast, a fewcycle pulse can generate electron wave packets moving in a single direction, as indicated in Fig. 10(f). Thus, a static field can reflect in principle the entire detached electron wave packet back to the origin, thereby doubling the potential modulation of the $\mathrm{H}^{-}$detachment probability (all other factors being equal). For the parameters considered below, fewcycle pulses can increase the modulation of the detachment probability (as compared to many-cycle pulses) well beyond this factor of 2 .

Consider therefore two nearly single-cycle pulses, each having a duration of $50 \mathrm{fs}$, a frequency equal to $\omega$ $\simeq 0.0046$ a.u., and a relative phase of $\pi$; the two pulses are shown in Figs. 12(a) and 12(b). The corresponding

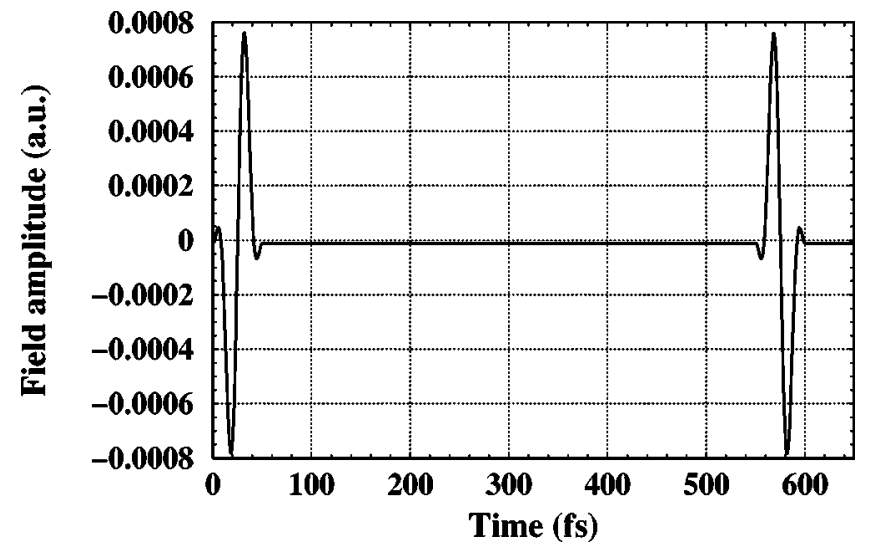

FIG. 13. Double single-cycle pulses in the presence of a static electric field $E_{s}=60 \mathrm{kV} / \mathrm{cm}$, with the second pulse phase shifted by $\pi$ and delayed by $\tau \simeq 550$ fs with respect to the first one. Each pulse has the same duration of $T=50 \mathrm{fs}$ and a maximum amplitude of $5 \times 10^{6} \mathrm{~V} / \mathrm{cm}$. All fields are polarized along the $z$ axis.

transition-probability densities are plotted in Figs. 12(c) and 12(d), respectively. From Fig. 12(c) we see that nearly all of the electrons produced by the laser pulse in Fig. 12(a) have negative momenta in the range $-0.4 \leqslant K_{z} \leqslant 0.0$ a.u., while those detached by the $\pi$-shifted pulse in Fig. 12(b), shown in Fig. 12(d), are ejected in the opposite direction with positive momenta primarily in the range $0 \leqslant K_{z} \leqslant 0.4$ a.u..

Thus, if a static field is applied to reflect the electron wave packet produced by the laser pulse in Fig. 12(a) back to the origin, then a time-delayed, $\pi$-phase shifted second pulse similar to that in Fig. 12(b) can be used to produce a second electron wave packet that overlaps the reflected one. Both wave packets will move in the same direction and will interfere either constructively or destructively with each other, depending on the time delay. Therefore, one can control the $\mathrm{H}^{-}$detachment probability by controlling the time delay between the two pulses.

To demonstrate coherent control of the $\mathrm{H}^{-}$detachment process, we combine the above single-cycle pulses [Figs. 12(a) and (b)] with a static electric field; the $\pi$-shifted second single-cycle pulse is delayed with respect to the first by a time $\tau$. The field of the combined single-cycle pulses is illustrated in Fig. 13 for the case of $\tau \simeq 550 \mathrm{fs}$. The electron wave packet produced by the first single-cycle pulse moves along the negative $K_{z}$ axis and is reflected by the potential of a negative static electric field $E_{s}$. Fig. 14 illustrates this reflection for the case of $E_{s}=-60 \mathrm{kV} / \mathrm{cm}$. In Fig. 14 the solid line represents the electron wave packet $\left|\Psi_{W P}\left(p_{z}, t\right)\right|^{2}[\mathrm{cf}$. Eq. (27)] generated by the first single-cycle pulse as a function of time $t$. For reference, the dashed line represents the electron wave packet that will be produced at some later time $\tau$ by the second ( $\pi$-shifted) laser pulse; this second wave packet is shown at fixed time $t=50 \mathrm{fs}$, i.e., at the end of the second pulse. One sees in Fig. 14 that at the end of the first single-cycle pulse (at $t=50 \mathrm{fs}$ ) the electron wave packet produced by this pulse (i.e., shown by the solid line) is moving to the left (i.e., with negative values $p_{z}$ ). As the time increases this wave packet is first slowed by the static field (i.e., located at smaller negative values of $p_{z}$ ), and then re- 

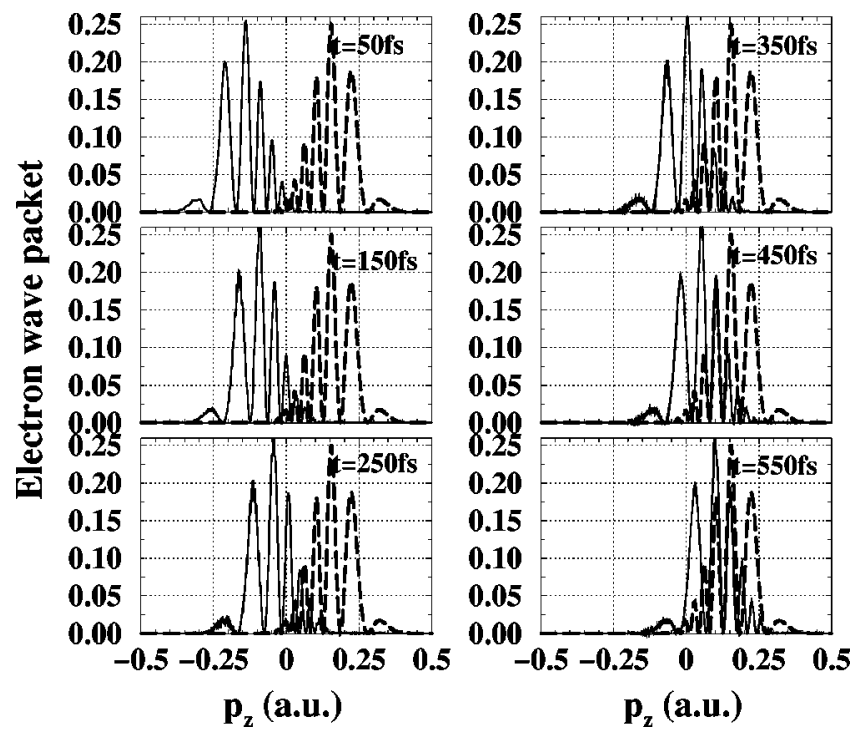

FIG. 14. The solid line in each panel shows the electron wave packet $\left|\Psi_{W P}\left(p_{x}=p_{y}=0, p_{z}, t\right)\right|^{2}$ produced by the first single-cycle pulse in Fig. 13 plotted vs $p_{z}$ for six values of time, $50 \mathrm{fs} \leqslant t$ $\leqslant 550$ fs. This wave packet is slowed and then reflected by the potential of a static electric field of strength $-60 \mathrm{kV} / \mathrm{cm}$. For reference, the electron wave packet produced by the second ( $\pi$-shifted) pulse shown in Fig. 13 is shown by the dashed line at the fixed time $t=50 \mathrm{fs}$ in each panel.

flected (i.e., taking on positive values of $p_{z}$ ). By the time $t$ $=550 \mathrm{fs}$ the electron wave packet produced by the first single-cycle pulse overlaps the region of momentum space occupied by the wave packet produced by the second singlecycle pulse. This means that if the second pulse is delayed with respect to the first by $t \geqslant 550 \mathrm{fs}$, then one can expect interference between the two wave packets, thus allowing one to control $\mathrm{H}^{-}$detachment by tuning the time delay $\tau$.

Using our generalized Keldysh-type formalism, we have calculated the total detachment probability $P$ [cf. Eq. (25)] for the above-described coherent control scheme as a function of the time delay $\tau$ between the two single-cycle pulses. Our results are shown in Fig. 15. One sees that by varying the time delay $\tau$ the total detachment probability exhibits Ramsey fringes [55,36,37] because the phase difference between the two electron wave packets is sensitive to the time delay between the two single-cycle pulses. The modulation, which is defined as the ratio of the change from maximum to minimum to the maximum detachment probability [23], attains a magnitude of nearly $30 \%$ for $\tau=640$ fs. (This time delay agrees with the prediction of a one-dimensional classical calculation for the electron reflection time for an electron detached by the first pulse.) This 30\% modulation of the $\mathrm{H}^{-}$detachment probability is far higher than the $10 \%$ modulation obtained in the analysis of Ref. [24] using many-cycle pulses and the same kind of coherent control scheme.

In Fig. 16 we show the transition-probability density $W(\mathbf{K})$ [cf. Eq. (24)] as a function of electron momentum $K_{z}$ for values of the time delay $\tau$ close to 640 fs. In Fig. 16(a), the time delay is equal to $\tau=636.2 \mathrm{fs}$. One sees that the electron wave packets interfere destructively in the momen-

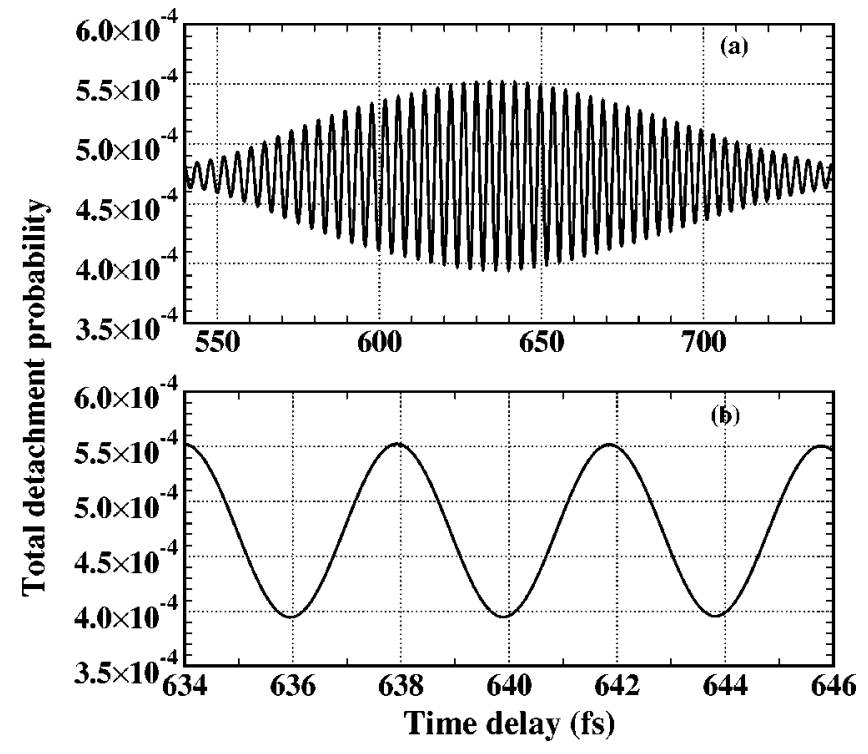

FIG. 15. The total detachment probability of $\mathrm{H}^{-}$for laser pulses of the form in Fig. 13 plotted as a function of the time delay $\tau$ between the two single-cycle pulses: (a) $540 \mathrm{fs} \leqslant \tau \leqslant 740 \mathrm{fs}$; (b) larger scale view of the region of the maximum in (a): $634 \mathrm{fs} \leqslant \tau$ $\leqslant 646$ fs.

tum range 0.18 a.u. $\leqslant K_{z} \leqslant 0.2$ a.u. In contrast, Fig. 16(b) shows constructive quantum interference in this same momentum region for the case $\tau=638.3$ fs. In Fig. 16(c) we plot the detachment probability density for momenta in the range 0.16 a.u. $\leqslant K_{z} \leqslant 0.22$ a.u. and laser-pulse separation in the range $632 \mathrm{fs} \leqslant \tau \leqslant 640 \mathrm{fs}$. One observes many oscillations in the probability density owing to constructive and destructive interferences. For values of $K_{z}$ outside the range shown in Fig. 16, the magnitudes of the oscillations in $W\left(K_{x}=K_{y}=0, K_{z}\right)$ decrease because the electron wave packets produced by the two laser pulses only partially overlap (see, e.g., Fig. 14). In general, as $\tau$ increases a particular peak in the pattern of $W\left(K_{x}=K_{y}=0, K_{z}\right)$ produced by the first laser pulse overlaps in succession with the different peaks in the pattern of $W\left(K_{x}=K_{y}=0, K_{z}\right)$ produced by the second laser pulse.

\section{Phase control of few-cycle pulses}

Unlike the situation of many-cycle long pulses, the absolute phase of a few-cycle pulse with respect to its temporal envelope turns out to be important $[6,25,56-60]$. In fact, the absolute phase of a few-cycle pulse determines the field configuration inside the pulse envelope. Thus, one may expect this phase to be an additional control parameter for $\mathrm{H}^{-}$detachment when a few-cycle laser pulse is employed. We demonstrate this fact in Fig. 17, which shows the $\mathrm{H}^{-}$detachment probability [cf. Eq. (25)] as a function of the absolute phase $\phi$ of the essentially single-cycle pulse shown in Fig. 12(a) for $\phi=0^{\circ}$ and in Fig. 12(b) for $\phi=180^{\circ}$. One sees that the $\mathrm{H}^{-}$detachment probability density varies between $\simeq 2.33 \times 10^{-4}$ and $\simeq 6.15 \times 10^{-4}$ as the phase of the pulse increases from $0^{\circ}$ to $360^{\circ}$. The modulation (i.e., the differ- 


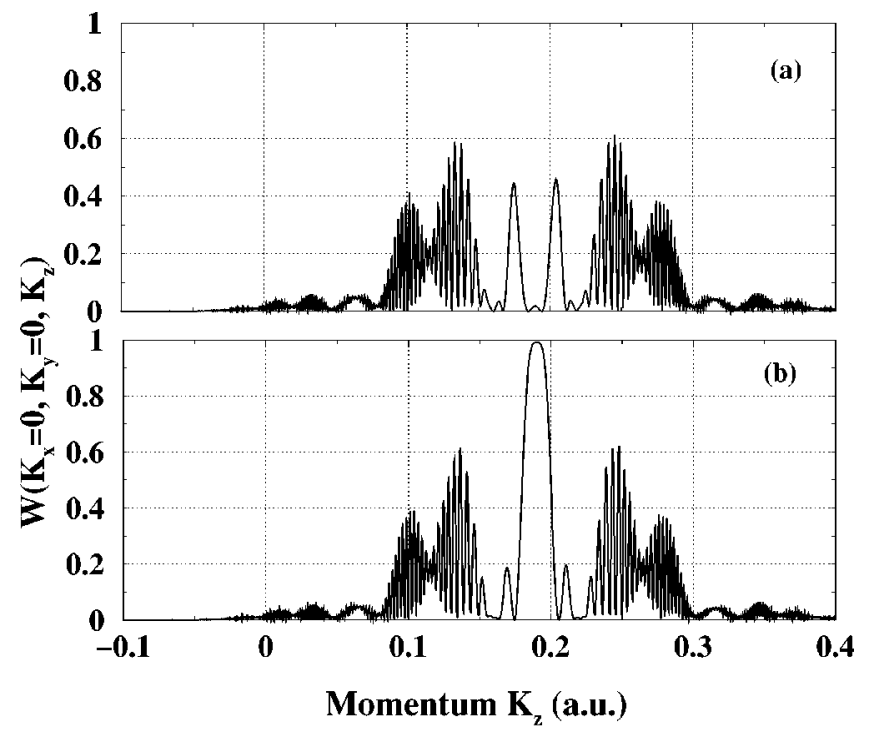

$\mathrm{W}\left(\mathrm{K}_{\mathrm{x}}=0, \mathrm{~K}_{\mathrm{y}}=0, \mathrm{~K}_{\mathrm{z}}\right)$

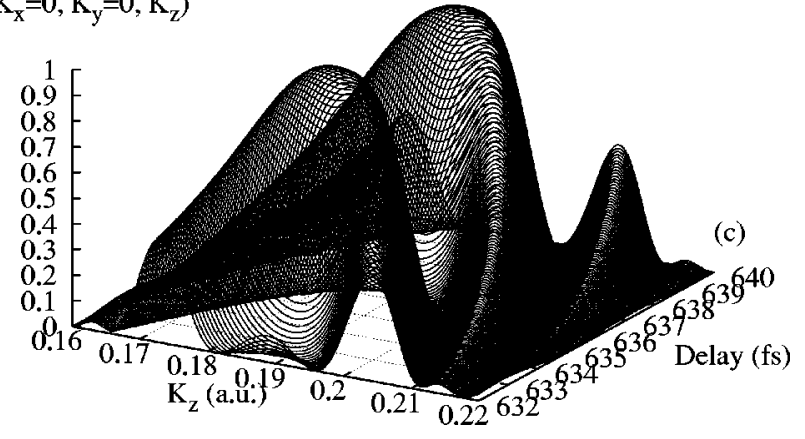

FIG. 16. $\mathrm{H}^{-}$detachment probability density $W\left(K_{x}=K_{y}\right.$ $=0, K_{z}$ ) for different time delays $\tau$ between the two single-cycle pulses in Fig. 13: (a) $\tau=636.2 \mathrm{fs}$; (b) $\tau=638.3 \mathrm{fs}$; (c) $632 \mathrm{fs} \leqslant \tau$ $\leqslant 640$ fs.

ence between the highest and lowest probabilities divided by the highest probability) achieved by varying $\phi$ is seen to be more than $60 \%$.

Consider now the dependence of this phase control on the laser-pulse duration and the laser frequency. In Fig. 18 we plot the $\mathrm{H}^{-}$detachment probability as a function of the absolute phase of the applied laser pulse for three different pulse durations: (a) $50 \mathrm{fs}$, (b) $150 \mathrm{fs}$, and (c) $300 \mathrm{fs}$. The laser frequency is $\omega \simeq 0.0046$ a.u. in each case.

One sees in Fig. 18 that, although the total detachment probability increases with increasing pulse duration, its modulation decreases dramatically from $\sim 62 \%$ in Fig. 18 (a) to $\sim 10 \%$ in Fig. 18(c).

In Fig. 19 we show the dependence of phase control of $\mathrm{H}^{-}$detachment on the laser frequency. For a laser-pulse duration of $50 \mathrm{fs}$, results for three different laser frequencies are shown: (a) $\omega \simeq 0.0046$ a.u., (b) $\omega \simeq 0.006$ a.u., and (c) $\omega$ $\simeq 0.01$ a.u. These results for changing the frequency for fixed pulse duration are similar to those shown in Fig. 18 for changing the pulse duration while keeping the frequency fixed. Namely, when the laser frequency increases, the magnitude of the detachment probability becomes larger, but its modulation as a function of the absolute phase turns out to be

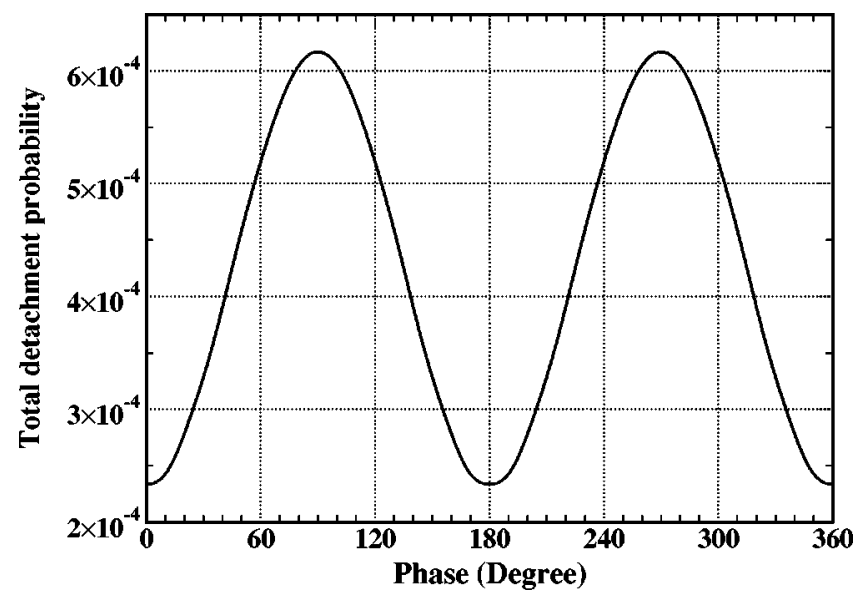

FIG. 17. $\mathrm{H}^{-}$detachment probability as a function of the absolute phase of a single-cycle pulse [shown in Fig. 12(a) for $\phi=0^{\circ}$ and in Fig. 12(b) for $\phi=180^{\circ}$ ]. The $\sin ^{2}$ pulse parameters are $T$ $=50$ fs and $\omega \simeq 0.0046$ a.u., as in Figs. 12(a) and 12(b).

smaller, varying from $\sim 62 \%$ in Fig. 19 (a) to $\sim 2.5 \%$ in Fig. 19(c).

In general, whenever a laser pulse becomes a "manycycle" pulse either by increasing the laser frequency (but keeping the same pulse duration) or by extending the pulse duration (but maintaining the same frequency), then one's ability to modulate the $\mathrm{H}^{-}$detachment probability by varying the absolute phase $\phi$ becomes fairly low. Clearly the absolute phase of the laser field with respect to the pulse envelope is critical only for few-cycle pulses.

Many researchers have discussed how to measure the absolute phase of a few-cycle pulse [61]. Here, our results showing that the total detachment probability is sensitive to the absolute phase of a few-cycle pulse imply a possible

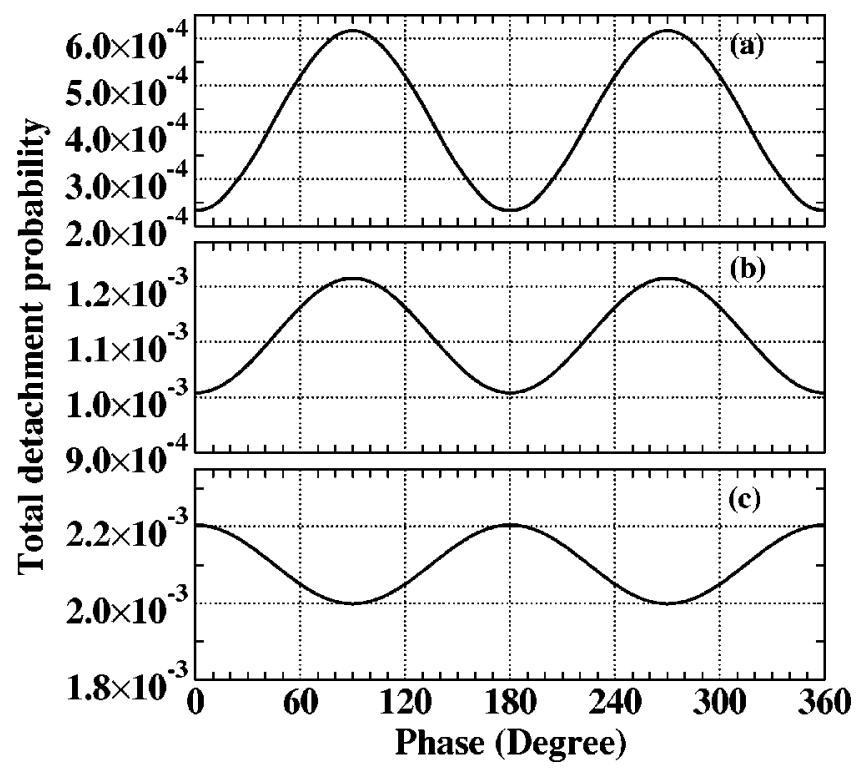

FIG. $18 . \mathrm{H}^{-}$detachment probability as a function of the absolute phase $\phi$ of the laser pulse for three different pulse durations $T$ : (a) $50 \mathrm{fs}$, (b) $150 \mathrm{fs}$, and (c) $300 \mathrm{fs}$. The laser frequency in each case is $\omega \simeq 0.0046$ a.u. [To facilitate comparison on the reduced amplitude scale used, (a) reproduces Fig. 17.] 


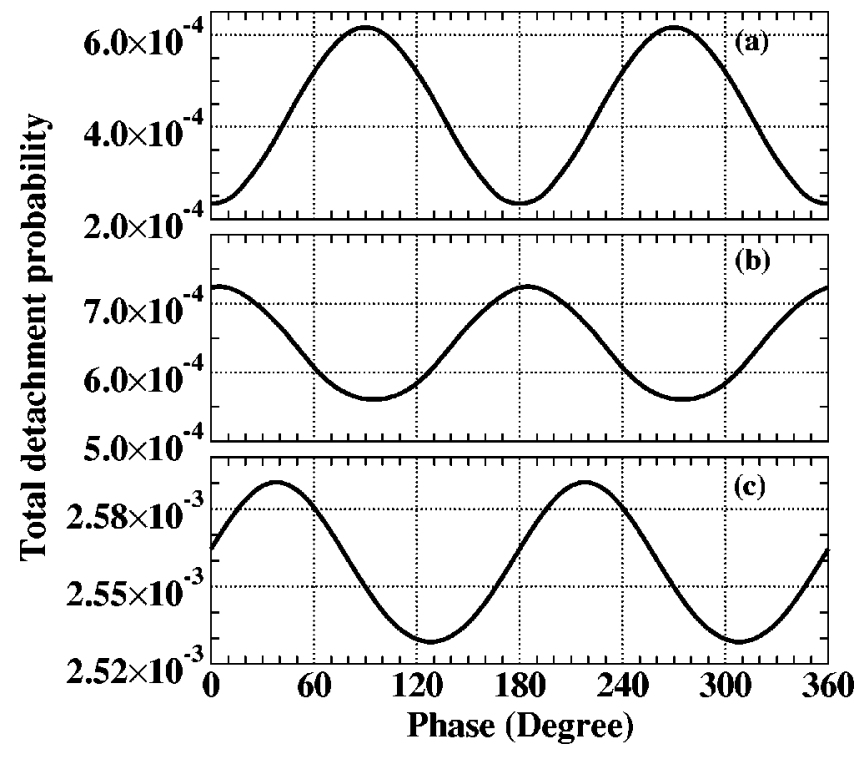

FIG. 19. $\mathrm{H}^{-}$detachment probability as a function of the absolute phase of the laser pulse for three different frequencies: (a) $\omega$ $\simeq 0.0046$ a.u., (b) $\omega \simeq 0.006$ a.u., and (c) $\omega \simeq 0.01$ a.u. The laser pulse duration is $50 \mathrm{fs}$ in each case. [To facilitate comparison on the reduced amplitude scale used, (a) reproduces Fig. 17.]

means to measure it. In particular, the pattern of the detached electron distribution as a function of $K_{z}$ depends sensitively on the absolute phase of a few-cycle pulse. This can be seen in Fig. 20. By analyzing the interference pattern of the detached electron spectrum in momentum space, one can associate a particular absolute phase of the few-cycle laser pulse with a particular pattern. [Recall that the single-cycle pulse produces an interference pattern that is similar to that produced by two half-cycle pulses, as shown in Fig. 6(c).] One sees that the pattern for any phase $\phi<\pi$ is a mirror image (with respect to the $K_{z}=0$ axis) of the pattern for the phase $\phi+\pi$. For $\phi=0$ (and $\phi=2 \pi$ ) there exist values of $K_{z}$ for which the probability is close to zero. For $0<\phi<\pi$ all values of $K_{z}$ are possible. For $\phi<\pi / 2$ (respectively $\phi>\pi / 2$ ) the "fine structure" of maxima and minima appear to the left (respectively to the right) of the peak of the probability distribution for $\phi=\pi / 2$. Also, for $\phi=\pi / 2$ and $3 \pi / 2$ the probability distribution has only ripples, but no deep minima. (There does appear to be a shallow minimum at the center of the distribution.)

\section{SUMMARY AND CONCLUSIONS}

In this work we have carried out and presented an extensive study of negative ion detachment using few-cycle pulses. Primarily for the purpose of being able to treat halfcycle pulses, we have extended the usual strong field, $S$-matrix theory treatment used extensively by others to treat the case in which the vector potential is nonzero at the end of the pulse. Our numerical and theoretical analyses of the halfcycle pulse detachment process, particularly for the case in which oppositely polarized half-cycle pulses are separated by a time delay, have elucidated the origin of the interference pattern seen in detachment by single-cycle pulses. We have
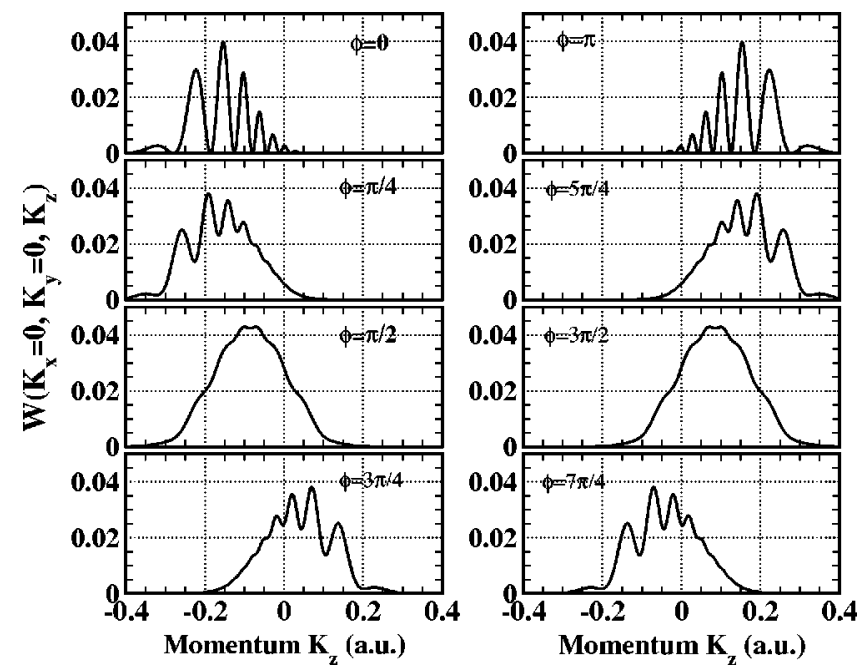

FIG. $20 . \mathrm{H}^{-}$detachment probability density by a 50 -fs singlecycle laser pulse having an amplitude $5 \times 10^{6} \mathrm{~V} / \mathrm{cm}$, a frequency $\omega \simeq 0.0046$ a.u., and the following phases $\phi$ with respect to its $\sin ^{2}$ temporal envelope: $\phi=0, \pi / 4, \pi / 2,3 \pi / 4, \pi, 5 \pi / 4,3 \pi / 2$, and $7 \pi / 4$. [Note that the laser pulse for $\phi=0$ is shown in Fig. 12(a); that for $\phi=\pi$ is shown in Fig. 12(b)].

also proposed a scheme for controlling negative ion detachment using two single-cycle pulses having opposite phases together with a weak static electric field (to reflect back to the origin the electron wave packet produced by the first single-cycle pulse). By use of this coherent control scheme, modulation of negative ion detachment (as a function of the time delay between the pulses) by about $30 \%$ has been demonstrated, which is far higher than predicted previously for this scheme with use of many-cycle pulses. The transition from single-, to few-, to many-cycle pulses has been analyzed as the laser frequency and the pulse duration are varied. Owing to the finite width of short-laser pulses, we have demonstrated that it is possible to have two-photon detachment probabilities of magnitudes comparable to those of single-photon detachment (when the tail of the frequency distribution of the pulse overlaps the one-photon threshold). Finally, we have shown the importance of the relative phase of the carrier wave relative to the pulse envelope for fewcycle pulses and the effects this phase has on detached electron momentum distributions. All of our predicted results were carried out for the $\mathrm{H}^{-}$negative ion; however, qualitatively our predictions apply quite generally to detachment by short pulses of any negative ion having $s$-state valence electrons. Our expectation is that similar results will obtain also for short-pulse detachment or ionization of any target species.

\section{ACKNOWLEDGMENTS}

The authors gratefully acknowledge useful discussions with Ilya I. Fabrikant, Mikhail V. Frolov, and Dejan B. Milošević. This work was funded in part by the National Science Foundation under Grant No. PHY-0070980. 
[1] M. Nisoli, S. De Silvestri, O. Svelto, R. Szipöcs, K. Ferencz, Ch. Spielmann, S. Sartania, and F. Krausz, Opt. Lett. 22, 522 (1997).

[2] T. Brabec and F. Krausz, Rev. Mod. Phys. 72, 545 (2000), and references therein.

[3] A. de Bohan, Ph. Antoine, D.B. Milošević, and B. Piraux, Phys. Rev. Lett. 81, 1837 (1998).

[4] H. Rabitz, R. de Vivie-Riedle, M. Motzkus, and K. Kompa, Science 288, 824 (2000).

[5] R.M. Potvliege, N.J. Kylstra, and C.J. Joachain, J. Phys. B 33, L743 (2000).

[6] G.G. Paulus et al., Nature (London) 414, 182 (2001).

[7] B.I. Greene, J.F. Federici, D.R. Dykaar, R.R. Jones, and P.H. Bucksbaum, Appl. Phys. Lett. 59, 893 (1991).

[8] D. You, R.R. Jones, D.R. Dykaar, and P.H. Bucksbaum, Opt. Lett. 18, 290 (1993).

[9] R.R. Jones, D. You, and P.H. Bucksbaum, Phys. Rev. Lett. 70, 1236 (1993).

[10] R.R. Jones, Phys. Rev. Lett. 76, 3927 (1996).

[11] J. Ahn, D.N. Hutchinson, C. Rangan, and P.H. Bucksbaum, Phys. Rev. Lett. 86, 1179 (2001).

[12] C. Rangan, K.J. Schafer, and A.R.P. Rau, Phys. Rev. A 61, 053410 (2000).

[13] K.J. LaGattuta and P.B. Lerner, Phys. Rev. A 49, R1547 (1994).

[14] K.J. LaGattuta, Phys. Rev. A 53, 1762 (1996).

[15] L.V. Keldysh, Zh. Éksp. Teor. Fiz. 47, 1945 (1964) [Sov. Phys. JETP 20, 1307 (1965)].

[16] F.H.M. Faisal, J. Phys. B 6, L312 (1973).

[17] H.R. Reiss, Phys. Rev. A 22, 1786 (1980).

[18] Bo Gao and A.F. Starace, Phys. Rev. A 42, 5580 (1990).

[19] W. Becker, S. Long, and J.K. McIver, Phys. Rev. A 42, 4416 (1990)

[20] W. Becker, S. Long, and J.K. McIver Phys. Rev. A 50, 1540 (1994).

[21] W. Becker, A. Lohr, M. Kleber, and M. Lewenstein, Phys. Rev. A 56, 645 (1997).

[22] G.F. Gribakin and M.Yu. Kuchiev, Phys. Rev. A 55, 3760 (1997).

[23] Qiaoling Wang and A.F. Starace, Phys. Rev. A 48, R1741 (1993).

[24] Qiaoling Wang and A.F. Starace, Phys. Rev. A 51, 1260 (1995).

[25] D.B. Milošević, G.G. Paulus, and W. Becker, Phys. Rev. Lett. 89, 153001 (2002).

[26] D.B. Milošević, G.G. Paulus, and W. Becker, Laser Phys. 13, 948 (2003).

[27] W.E. Lamb, R.R. Schlicher, and M.O. Scully, Phys. Rev. A 36, 2763 (1987); see especially Appendix B.

[28] C. Cohen-Tannondji, J. Dupont-Roc, and G. Grynberg, Photons and Atoms: Introduction to Quantum Electrodynamics (Wiley, New York, 1989), pp. 273 and 274.

[29] E.G. Bessonov, Zh. Eksp. Teor. Fiz. 80, 852 (1981) [Sov. Phys. JETP 53, 433 (1981)].

[30] R. Grobe and M.V. Fedorov, Laser Phys. 3, 165 (1993).

[31] B. Rau, T. Tajima, and H. Hojo, Phys. Rev. Lett. 78, 3310 (1997); 84, 3211 (2000); K.J. Kim, K.T. McDonald, G.V. Stupakov, and M.S. Zolotorev, ibid. 84, 3210 (2000).

[32] L.B. Madsen, Phys. Rev. A 65, 053417 (2002).
[33] M. Shapiro and P. Brumer, Adv. At., Mol., Opt. Phys. 42, 287 (2000), and references therein.

[34] N.L. Manakov, V.D. Ovsiannikov, and A.F. Starace, Phys. Rev. Lett. 82, 4791 (1999).

[35] Z.-M. Wang and D.S. Elliott, Phys. Rev. Lett. 87, 173001 (2001).

[36] G. Alber, H. Ritsch, and P. Zoller, Phys. Rev. A 34, 1058 (1986).

[37] L.D. Noordam, D.I. Duncan, and T.F. Gallagher, Phys. Rev. A 45, 4734 (1992).

[38] I.Yu. Kiyan and H. Helm, Phys. Rev. Lett. 90, 183001 (2003).

[39] N.L. Manakov, M.V. Frolov, A.F. Starace, and I.I. Fabrikant, J. Phys. B 33, R141 (2000).

[40] Yu. N. Demkov and V. N. Ostrovskii, Zero-Range Potentials and Their Applications in Atomic Physics (Plenum, New York, 1988).

[41] R. R. Schlicher, W. Becker, J. Bergou, and M. O. Scully, in Quantum Electrodynamics and Quantum Optics, edited by A. O. Barut (Plenum, New York, 1984) pp. 405-441; W. Becker, Opt. Commun. 56, 107 (1985); W.E. Lamb, Jr., R.R. Schlicher, and M.O. Scully, Phys. Rev. A 36, 2763 (1987).

[42] D. Guo, T. Ảberg, and B. Crasemann, Phys. Rev. A 40, 4997 (1989).

[43] R. Kopold, W. Becker, and M. Kleber, Opt. Commun. 179, 39 (2000).

[44] S.P. Goreslavskii, S.V. Popruzhenko, R. Kopold, and W. Becker, Phys. Rev. A 64, 053402 (2001); [note that there is a misprint in Eq. (3): the limits on the integral should be omitted.]

[45] T. Ohmura and H. Ohmura, Phys. Rev. 118, 154 (1960).

[46] M.L. Du and J.B. Delos, Phys. Rev. A 38, 5609 (1988).

[47] C. Cohen-Tannondji, J. D. Dupont-Roc, and G. Grynberg, Atom-Photon Interactions (Wiley, New York, 1992), p. 33, Eq. (38).

[48] Yu.N. Demkov and G.F. Drukarev, Zh. Éksp. Teor. Fiz. 47, 918 (1964) [Sov. Phys. JETP 20, 614 (1965)].

[49] S.N. Kaplan, G.A. Paulikas, and R.V. Pyle, Phys. Rev. 131, 2574 (1963).

[50] M.V. Frolov, N.L. Manakov, B. Borca, and A.F. Starace, J. Phys. B 34, L579 (2001).

[51] M.V. Ammosov, N.B. Delone, and V.P. Krainov, Zh. Eksp. Teor. Fiz. 91, 2008 (1986) [Sov. Phys. JETP 64, 1191 (1986)].

[52] B.E. Tannian et al., Phys. Rev. A 62, 043402 (2000).

[53] B. Borca, M.V. Frolov, N.L. Manakov, and A.F. Starace, Phys. Rev. Lett. 87, 133001 (2001).

[54] N.L. Manakov, M.V. Frolov, B. Borca, and A.F. Starace, J. Phys. B 36, R49 (2003).

[55] N.F. Ramsey, Phys. Rev. 78, 695 (1950).

[56] E. Cormier and P. Lambropoulos, Eur. Phys. J. D 2, 15 (1998).

[57] G. Tempea, M. Geissler, and T. Brabec, J. Opt. Soc. Am. B 16, 669 (1999).

[58] A. de Bohan, P. Antoine, D.B. Milošević, and B. Piraux, Phys. Rev. Lett. 81, 1837 (1998); Laser Phys. 9, 175 (1999).

[59] G.L. Yudin and M.Yu. Ivanov, Phys. Rev. A 64, 013409 (2002).

[60] S. Chelkowski and A.D. Bandrauk, Phys. Rev. A 65, 061802(R) (2002).

[61] P. Dietrich, F. Krausz, and P.B. Corkum, Opt. Lett. 25, 16 (2000); A. Apolonski et al., Phys. Rev. Lett. 85, 740 (2000). 